\title{
Distortion Isomerism in the Chemistry of Heterometallic Tin Clusters
}

\author{
Milan Melník ${ }^{\mathrm{a}}$, Ján Garaj ${ }^{\mathrm{a}}$, Aladár Valent ${ }^{\mathrm{b}}$ and Mária Kohútová \\ a'Department of Inorganic Chemistry, Slovak Technical University, Radlinskeho 9, 81237 \\ Bratislava, Slovak Republic \\ ${ }^{\mathrm{b}}$ Department of Chemical Theory of Drugs, Faculty of Pharmacy, Comenius University, \\ 83232 Bratislava, Slovak Republik
}

\begin{abstract}
The heterometallic tin clusters cover a huge field, as shown by a recent survey covering the crystallographic and structural data of over six hundred examples. About $8 \%$ of these clusters exist as isomers and are summarised in this review. The isomers are examples of distortion isomerism. These are discussed in terms of the coordination about the tin as well as heterometal atoms, and correlations are drawn between the donor atom, bond length and bond angles, with attention to distortion of the respective geometry.

Distortion isomers, differing only by degree of distortion in $M-L$ and $L-M-L$ angles, are the most common.
\end{abstract}

Keywords: Heterometal, Tin, Distortion, Isomerism

\section{CONTENTS}

0 ABBREVIATIONS

1. INTRODUCTION

2. DISTORTION ISOMERISM

2.1 Isomeric forms

2.2 Independent molecules

2.2.1 Dimers

2.2.2 Trimers

2.2.3 Tetramers

2.2.4 Oligomers

3. CONCLUSIONS

4. ACKNOWLEDGEMENTS

5. REFERENCES

\section{ABBREVIATIONS}

$\begin{array}{ll}\text { Ampy } & \text { 2-amino-6-methylpyridine } \\ \mathrm{Bdpp}^{\mathrm{t}} & \text { 2,4-bis(diphenylphosphino)pentane } \\ \mathrm{Bu}^{\mathrm{t}} & \text { tert-butyl }\end{array}$




\begin{tabular}{|c|c|}
\hline $\mathrm{Bu}_{2}^{\prime}$ bpy & 4,4'-di-tert-butyl-2,2'-bipyridine \\
\hline $\operatorname{cod}$ & 1,5 - cyclooctadiene \\
\hline cp & cyclopentadienyl \\
\hline cp* & pentamethylcyclopentadienyl \\
\hline cryp & cryptate \\
\hline db & diborolenyl \\
\hline 1,4-dio & 1,4-dioxane \\
\hline dme & 1,2-dimethoxyethane \\
\hline fu & furyl \\
\hline hx & hexagonal \\
\hline m & monoclinic \\
\hline $\mathrm{Me}$ & methyl \\
\hline mes & mesityl \\
\hline $\mathrm{m}-\mathrm{MeC}_{6} \mathrm{H}_{4}$ & meta-methylphenyl \\
\hline $\mathrm{np}_{3}$ & tris(2-diphenylphosphinoethyl) amine \\
\hline $\mathrm{NEt}_{4}$ & tetraethylammonium \\
\hline or & orthorhombic \\
\hline $\mathrm{Ph}$ & phenyl \\
\hline Pmdeta & $\left(\mathrm{MeNCH}_{2} \mathrm{CH}_{2}\right)_{2} \mathrm{NMe}$ \\
\hline $\mathrm{PMe}_{3}$ & trimethylphosphine \\
\hline $\operatorname{PPr}_{3}{ }^{i}$ & tri-isopropylphosphine \\
\hline $\operatorname{tg}$ & tetragonal \\
\hline thf & tetrahydrofuran \\
\hline tol & toluene \\
\hline tpsp & tri-isopropylsilylphosphanide \\
\hline tpspi & tri-isopropylsilylphosphandiide \\
\hline tr & triclinic \\
\hline
\end{tabular}

\section{INTRODUCTION}

Stereoselectivity in coordination compounds is very often related to important stereospecifity of biological systems, catalysis and stereochemical effects in technical processes. Isomers can be broadly classified into two major categories, structural and stereoisomers. The former can be divided into coordination, hydrate, ionization, linkage and polymerization sub-categories, and the latter can be divided into geometric (cis/trans, fac/mer), optical and distortion isomerism. Analysis of crystallographic and structural data of isomers of organotin compounds showed $/ 1,2 /$ that there are four types of isomerisms: distortion, cis-trans, ligand and $n$-mer, of which the first one prevails by far.

The large area of heterometallic tin clusters has recently been surveyed $/ 1,2 /$ with over 50 isomeric examples noted. In this review we analyse and classify these examples to show that only stereoisomersdistortion isomers were found and discussed. 


\section{DISTORTION ISOMERISM}

The coexistence of two or more species differing only by degree of distortion of $M-L$ bond distances and $\mathrm{L}-\mathrm{M}-\mathrm{L}$ bond angles is typical of the general class of distortion isomers $/ 4 /$. These are almost fifty such examples in the chemistry of di- and polymeric heterometallic tin compounds.

\subsection{Isomeric forms}

There are five examples which exist in two isomeric forms, and their structural parameters are gathered in Table 1. A potassium complex $\mathrm{Sn}\left(\mu-\mathrm{OSiPh}_{3}\right)_{3} \mathrm{~K}(\mathrm{dme})_{2} / 6 /$ exists in two different crystalline forms, monoclinic and triclinic. Both share the presence of a K-Sn unit bridged by three $\mu$-OSiPh ${ }_{3}$ anions. The forms differ by the presence of two bidentate $\mathrm{MeOC}_{2} \mathrm{H}_{4} \mathrm{OMe}(\mathrm{dme})$ ligands in the monomeric form, but only one bidentate in the triclinic form, the other being monodentate. In fact, the triclinic form contains two crystallographically independent molecules, both of formula $\operatorname{Sn}\left(\mu-\mathrm{OSiPh}_{3}\right)_{3} \mathrm{~K}\left(\eta^{2}-\mathrm{dme}\right)\left(\eta^{1}\right.$-dme). All three molecules are very similar in the $\mathrm{Sn}\left(\mathrm{OSiPh}_{3}\right)_{3} \mathrm{~K}$ sub-structure. Superimposed drawings reveal that the oxygen of the $\eta^{1}$-dme adopts a location between those of the two oxygens of one $\eta^{2}$-dme in the monoclinic form. The Sn - K distances differ only slightly between the monoclinic (3.515 $\AA)$ and triclinic forms (3.460 and 3.480 $\AA$ ) and rule out any direct metal - metal bond.

Two yellow SnPt dimers, triclinic and monoclinic $/ 7 /$, contain cis- $\left[\mathrm{Cl}_{2} \mathrm{Pt}\left(\mathrm{SnCl}_{3}\right)_{2}\right]^{-}$anions disordered in such a way as to be pseudo - centrosymmetric. This disordering involves half of the occupied $\mathrm{PtCl}_{2}$ sites appearing on either side of the centre. Simultaneously, one chlorine from each $\mathrm{SnCl}_{3}$ unit changes side while the other chlorine atoms appear in average positions with very small displacements between their positions. The Pt - Sn bond distance is $2.356(1) \AA$ (average), and $\mathrm{Sn}-\mathrm{Pt}-\mathrm{Sn}$ angle is $111.48(3)^{\circ}$. Unfortunately, data are available only for the triclinic form.

The colourless $\left[(\text { tol }) \mathrm{SnClAlCl}_{4}\right]_{2}$ derivative $/ 8 /$ exists in two triclinic isomeric forms ( $\alpha$ - and $\beta-$ ) differing mostly by degree of distortion. The two $\left(\eta^{6}-\right.$ tol $) S n$ units of the dimer are linked by two direct chlorine bridges and two 1,3-bidentate $\mathrm{AlCl}_{4}$ groups. The coordination of the $\mathrm{Sn}(\mathrm{II})$ centres is thus pseudo-tetrahedral (Table 1).

There are two dark red (monoclinic and triclinic) $\mathrm{SnRu}_{3}$ isomers $/ 9 /$ which are isostructural, one of them being shown in Fig. 1. The cluster consists of a triangular array of ruthenium atoms triply bridged by the (ampy) ligand, with two short edges $(R u-R u=2.878(1)$ and $2.695(1) \AA)$. These are also bridged by hydrido and diphenylalkenyl ligands, respectively. The $\mu_{3}, \eta^{2}$ - ampy ligand occupies three axial coordination sites, being linked to one $\mathrm{Ru}$ atom via the pyridinic nitrogen atom and to the other two $\mathrm{Ru}$ atoms by the exocyclic $\mathrm{N}$ atom. $\mathrm{A} \mathrm{SnPh}_{3}$ group is coordinated at an equatorial position to the first $\mathrm{Ru}$ atom being nearly cis to the hydride ligand. The cluster coordination shell is completed by seven terminal $\mathrm{CO}$ groups, three in axial sites trans to the (ampy) $\mathrm{N}$ atoms and four in equatorial sites. The $\mathrm{Sn}-\mathrm{Ru}$ bond distance of 2.623(1) $\AA$ in the monoclinic forms is about $0.039 \AA$ shorter than that found in the triclinic form (2.662(1) $\AA$. 


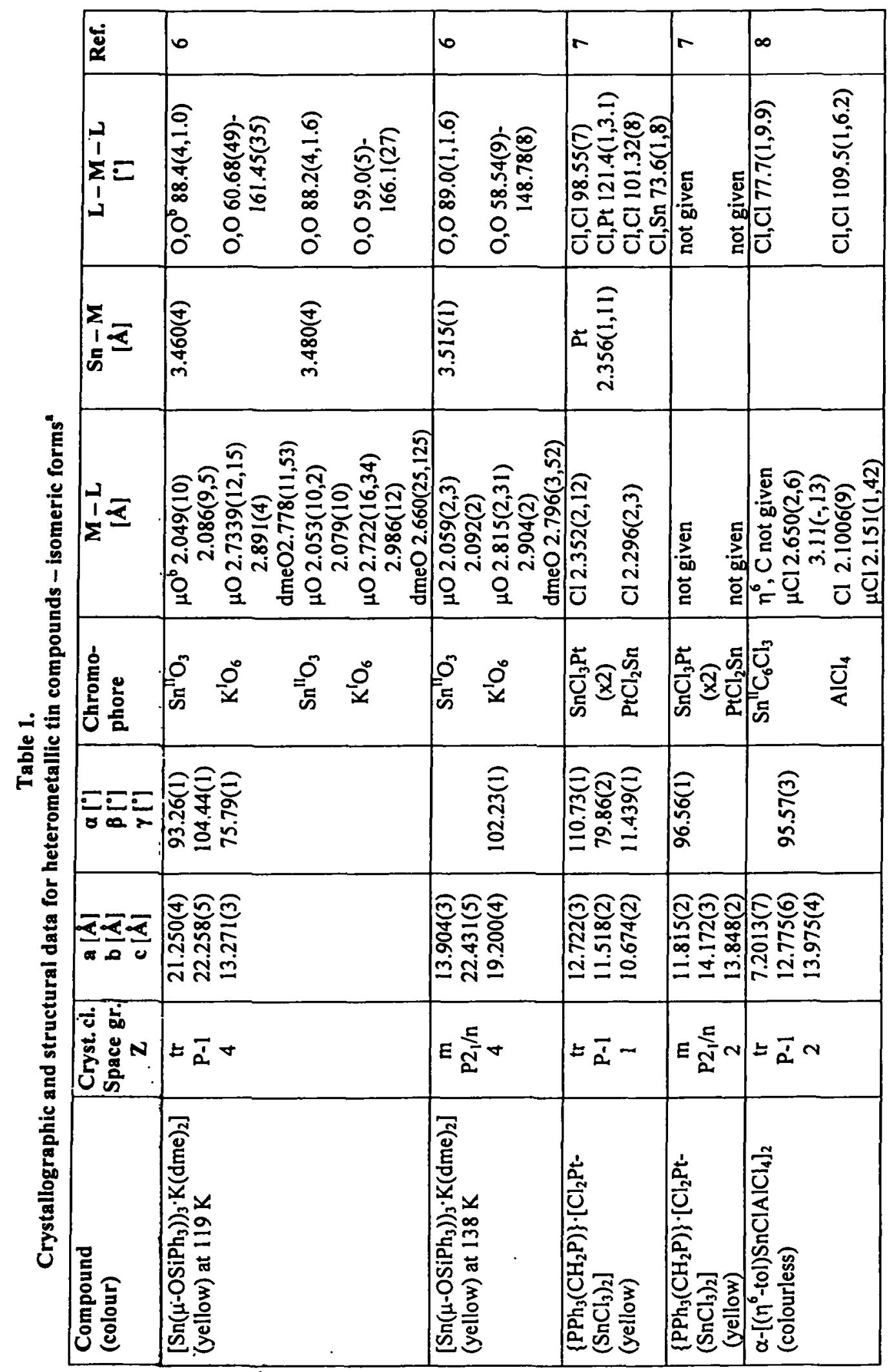




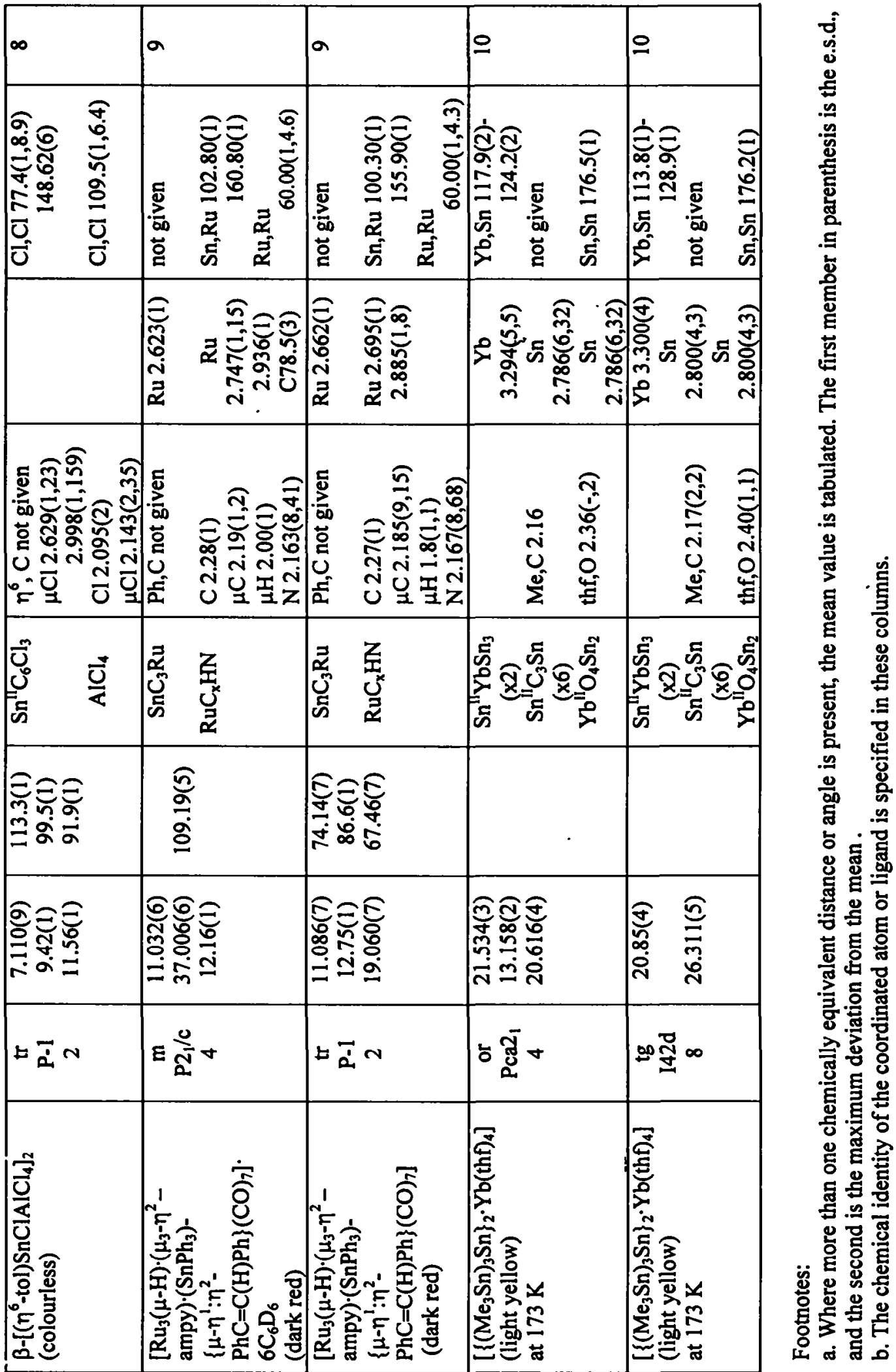




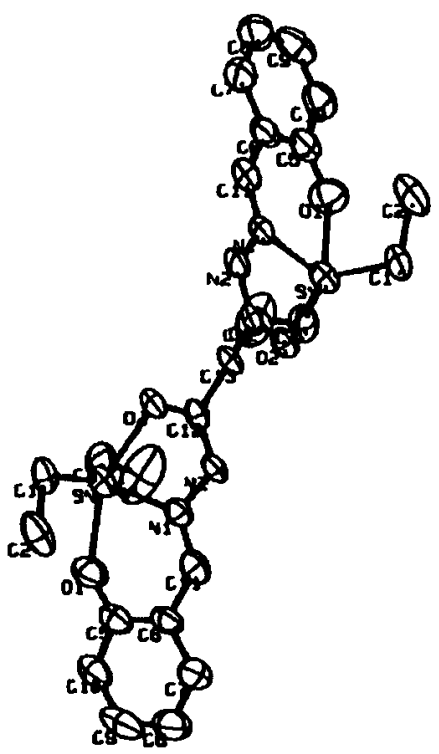

Fig. 1: Structure of $\mathrm{Ru}_{3}(\mathrm{H})(\mathrm{ampy})\{\mathrm{PhC}=\mathrm{C}(\mathrm{H}) \mathrm{Ph}\}\left(\mathrm{SnPh}_{3}\right)(\mathrm{CO})_{7} / 9 /$

There are two coloured (orthorhombic and tetragonal) $\mathrm{Sn}_{8} \mathrm{Yb}$ isostructural clusters $/ 10 /$. The molecules of the clusters have a distorted octahedral structure in which the central $\mathrm{Yb}$ atom is bonded to four $\mathrm{O}$ atoms of (thf) molecule equatorially and two $\mathrm{Sn}(\mathrm{II})$ atoms of two $\mathrm{Sn}\left(\mathrm{SnMe}_{3}\right)_{3}$ moieties axially (Fig. 2). The $\mathrm{Sn}-\mathrm{Yb}-$ Sn bond angles are $178.5(1)^{\circ}$ (orthorhombic) and 176.20(1) (tetragonal). The average $\mathrm{Yb}-\mathrm{Sn}$ distances are $3.294(5)^{\circ}$ (orthorhombic) and 3.300(4) $\AA$ (tetragonal). Two $\mathrm{Sn}(\mathrm{II})$ atoms in each cluster contain the $\mathrm{SnYbSn}_{3}$ chromophore with average $\mathrm{Sn}$ - Sn distances of 2.786(6) and 2.800(4) $\AA$, respectively. The remaining six $\mathrm{Sn}(\mathrm{II})$ atoms in each of the respective clusters contain the $\mathrm{SnC}_{3} \mathrm{Sn}$ chromophore.

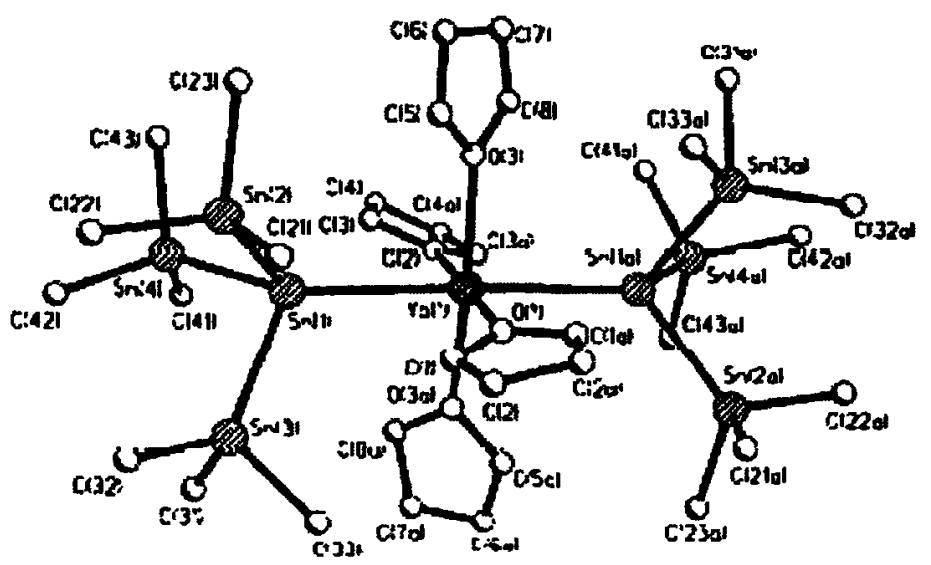

Fig. 2: Structure of $\left[\left\{\left(\mathrm{Me}_{3} \mathrm{Sn}\right)_{3} \mathrm{Sn}\right\}_{2} \mathrm{Yb}(\text { thf })_{4}\right] \quad / 10 /$ 


\subsection{Independent molecules}

There are forty heterometallic tin complexes which contain a crystallographically independent molecules within the same crystal /11-49/, and their structural parameters are gathered in Table 2.

\subsubsection{Dimers}

Seventeen derivatives are heterodimer tin complexes (Table $2 \mathrm{~A}$ ). A pale yellow $\mathrm{Ph}_{3} \mathrm{SnMn}(\mathrm{CO})_{5} / 11 /$ is the only example which contains four crystallographically independent molecules. In each of the molecules, $\mathrm{Ph}_{3} \mathrm{Sn}$ and $\mathrm{Mn}(\mathrm{CO})_{5}$ moieties are held together by a direct $\mathrm{Sn}-\mathrm{Mn}$ bond of 2.670(7) $\AA$ (molecule 1), 2.672(7) $\AA$ (molecule 2), 2.676(7) $\AA$ (molecule 3), and 2.678(7) $\AA$ (molecule 4). Each tin atom has pseudotetrahedral geometry $\left(\mathrm{SnC}_{3} \mathrm{Mn}\right.$ ). The sum of $\mathrm{Sn}-\mathrm{C}(\mathrm{x} 3)$ plus $\mathrm{Sn}-\mathrm{Mn}$ bond distances are $11.25 \AA$ (molecule 1), $11.23 \AA$ (molecule 2), $11.37 \AA$ (molecule 3), and 11.20 $\AA$ (molecule 4). The maximum deviation from the ideal tetrahedral bond angle $\left(109.4^{\circ}\right)$ is $4.8^{\circ}, 5.6^{\circ}, 5.4^{\circ}$ and $5.2^{\circ}$, respectively.

The remaining sixteen dimers contain two crystallographically independent molecules. In two colourless $\mathrm{Ph}_{3} \mathrm{SnLi}\left(\eta^{3}\right.$-pmdeta) $/ 12 /$ and [ $\left.\left\{\mathrm{SiMe}_{3} \mathrm{~N}\left(4-\mathrm{CH}_{3} \mathrm{C}_{6} \mathrm{H}_{4}\right)\right\}_{3} \mathrm{Sn} \cdot \mathrm{Li}(\text { thf })_{3}\right] / 13 /$, two nonequivalent moieties, $\mathrm{Ph}_{3} \mathrm{Sn}$ and $\mathrm{Li}\left(\eta^{3}\right.$-pmdeta) in the former and $\left\{\mathrm{SiMe}_{3} \mathrm{~N}\left(4-\mathrm{CH}_{3} \mathrm{C}_{6} \mathrm{H}_{4}\right)\right\}_{3} \mathrm{Sn}$ and $\mathrm{Li}(\text { thf })_{3}$ in the latter, are held together by direct $\mathrm{Sn}-\mathrm{Li}$ bond distances with the values of 2.861(7) and 2.882(7) $\AA / 12 /$ and 2.89(4) and 2.97(5) $\AA / 13 /$. Each $\mathrm{Sn}(\mathrm{II})$ as well as $\mathrm{Li}(\mathrm{I})$ atom is tetrahedrally coordinated, with the chromophores, $\mathrm{SnC}_{3} \mathrm{Li}$ and $\mathrm{LiN}_{3} \mathrm{Sn}$ $/ 12 /$ and $\mathrm{SnN}_{3} \mathrm{Li}$ and $\mathrm{LiO}_{3} \mathrm{Sn} / 13 /$ (Table 2A). The sum values of $\mathrm{Sn}-\mathrm{X}(\mathrm{x} 3)(\mathrm{X}=\mathrm{C} / 12 /$ or N/13/) and Sn Li bond distances are $9.50 \AA$ (molecule 1) and $9.47 \AA$ (molecule 2) /12/, and $9.33 \AA$ (molecule 1) and $9.39 \AA$ (molecule 2) /13/.

There are two colourless isostructural derivatives, $\mathrm{Ph}_{3} \mathrm{SnGeMe}_{3} / 14 /$ and $\mathrm{Ph}_{3} \mathrm{SnPbMe}_{3} / 15 /$, which contain two crystallographically independent molecules. In each of them two non-equivalent $\left(\mathrm{Ph}_{3} \mathrm{Sn}\right.$ and $\mathrm{GeMe}_{3} / 14 /$, $\mathrm{Ph}_{3} \mathrm{Sn}$ and $\left.\mathrm{PbPh}_{3} / 15 /\right)$ moieties are held together by a direct $\mathrm{Sn}-\mathrm{M}(\mathrm{M}=\mathrm{Ge}$ or $\mathrm{Pb})$ bond with the lengths of $\mathrm{Sn}-\mathrm{Ge}=2.567(6)$ and 2.596(4) $\AA$; and $\mathrm{Sn}-\mathrm{Pb}=2.809(2) \AA$ and 2.848(2) $\AA$. The average $\mathrm{Sn}-\mathrm{Ge}$ bond distance of $2.58 \AA$ is about $0.25 \AA$ shorter than that of $\mathrm{Sn}-\mathrm{Pb}$ bond distance $(2.83 \AA$ ), which corresponds well with the covalent radii of the respective $\mathrm{M}$ atoms ( $\mathrm{Ge}=1.22 \AA$ and $\mathrm{Pb}=1.47 \AA$ ). Each metal atom is tetrahedally coordinated $\left(\mathrm{SnC}_{3} \mathrm{M}\right.$ vs $\mathrm{MC}_{3} \mathrm{Sn}$ ) with different degree of distortion (Table $2 \mathrm{~A}$ ).

A green derivative /16/ has $\mathrm{Ph}_{3} \mathrm{Sn}$ and $\mathrm{Ticp}_{2} \mathrm{Cl}$ moieties held together by a direct $\mathrm{Sn}(\mathrm{II})-\mathrm{Ti}(\mathrm{IV})$ bond of 2.843(1) $\AA$. Each $\mathrm{Sn}(\mathrm{II})$ atom is tetrahedrally coordinated $\left(\mathrm{SnC}_{3} \mathrm{Ti}\right)$. The sum of all four bond distances ( $\mathrm{Sn}-$ $\mathrm{C}(\mathrm{x} 3)$ ) plus $\mathrm{Sn}-\mathrm{Ti}$ ) is $9.374 \AA$ (molecule 1 ) and $9.347 \AA$ (molecule 2) with the bond angles (C - Sn - C, C - $\mathrm{Sn}-\mathrm{Ti}$ ) ranging from 101.8(2) to $120.6(2)^{\circ}$ (molecule 1 ) and from 102.4(2) to $117.3(2)^{\circ}$ (molecule 2 ). The molecule 1 is somewhat less crowded, with a higher degree of distortion than the molecule 2.

A yellow $\mathrm{Me}_{3} \mathrm{SnMo}(\mathrm{H}) \mathrm{cp}_{2} / 17 /$, which also contains two crystallographically independent molecules, has a similar structure to that of $\mathrm{Ph}_{3} \mathrm{SnTicp}_{2} \mathrm{Cl} / 16 /$. Two non - equivalent $\left(\mathrm{Ph}_{3} \mathrm{Sn}\right.$ and $\left.\mathrm{Mo}(\mathrm{H}) \mathrm{cp}_{2}\right)$ moieties are held together by a direct $\mathrm{Sn}-$ Mo bond (2.755(1) and 2.758(1) $\AA$ (Table 2A).

In another SnMo derivative /18/ also, a direct Sn - Mo bond (2.508(3) $\AA$ (molecule 1) and 2.517(3) $\AA$ (molecule 2) connects two non - equivalent ((mes) $)_{2} \mathrm{Sn}$ and $\left.\mathrm{Mo}(\mathrm{CO})_{2}(\mathrm{cpMe})\right)$ moieties. 


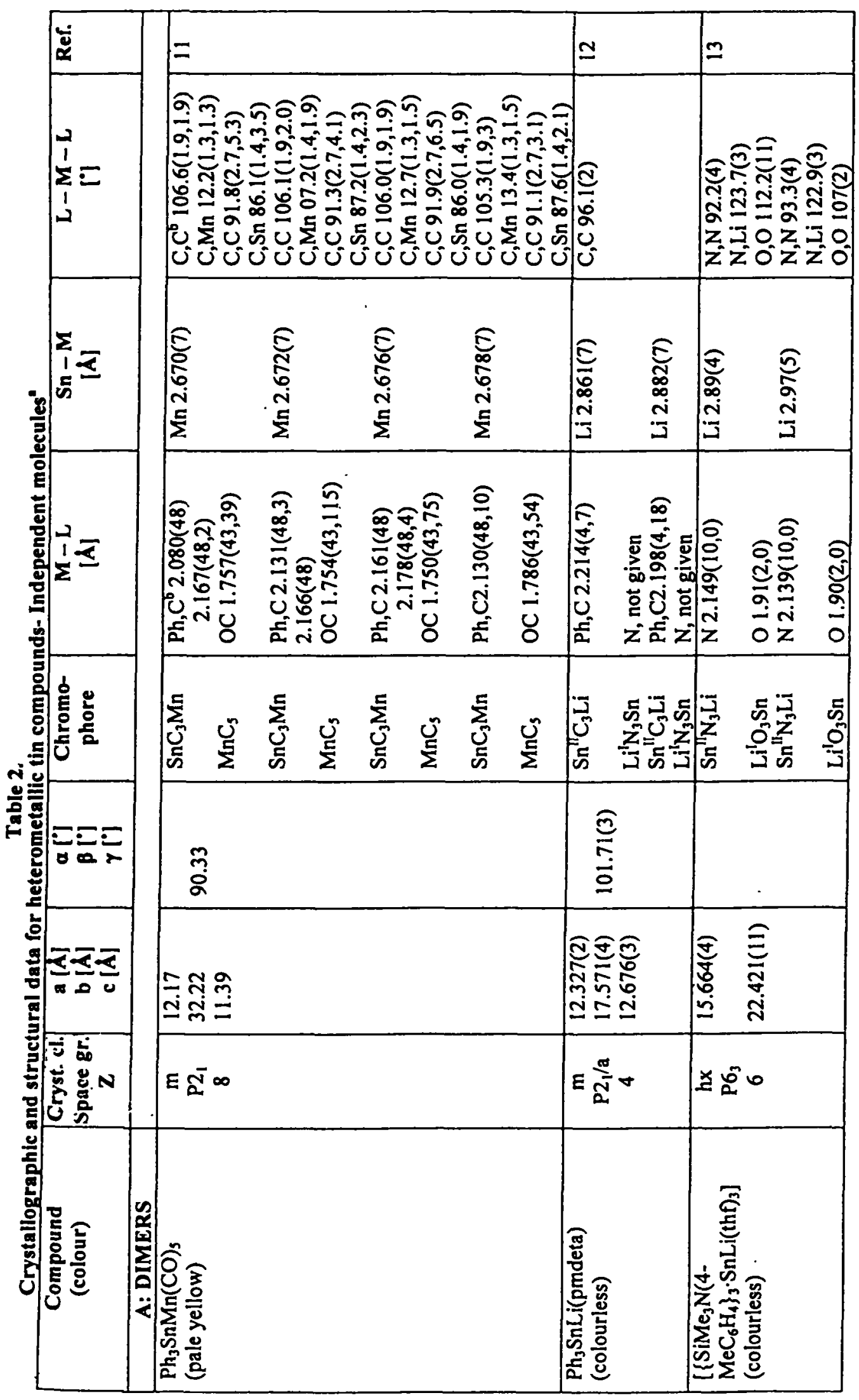




\begin{tabular}{|c|c|c|c|c|c|}
\hline 0 & & \pm & & $\cong$ & 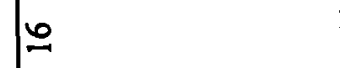 \\
\hline 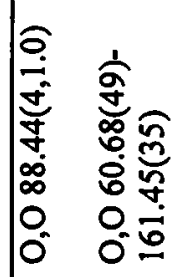 & 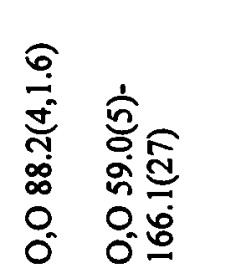 & 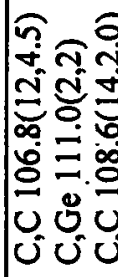 & 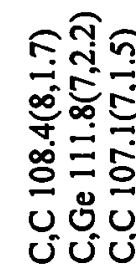 & 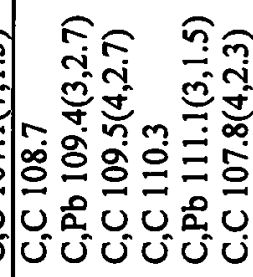 & 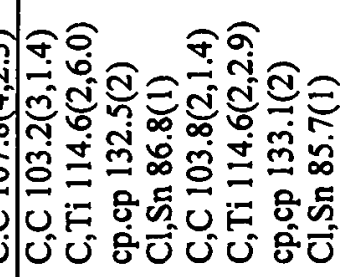 \\
\hline 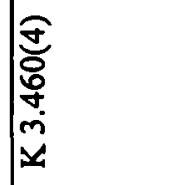 & 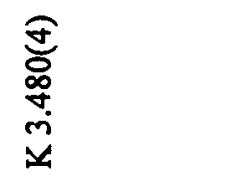 & 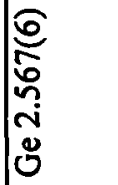 & 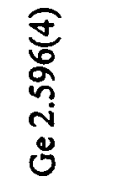 & 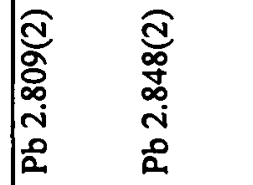 & 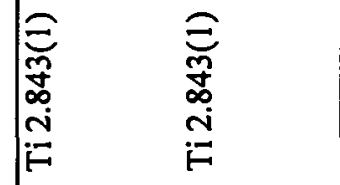 \\
\hline 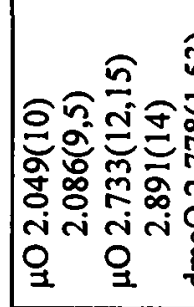 & 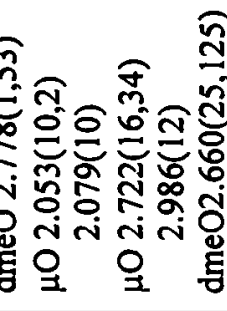 & 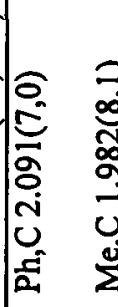 & 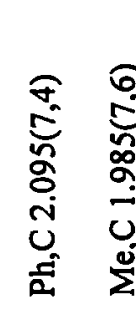 & 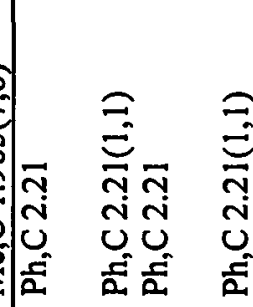 & 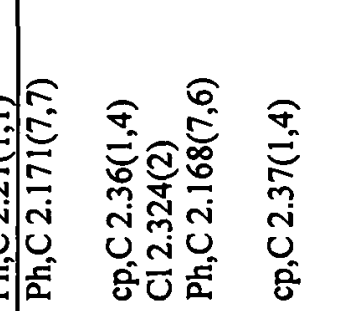 \\
\hline 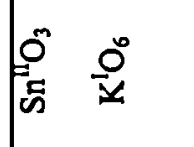 & 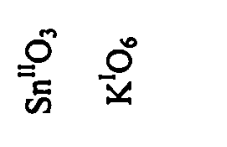 & 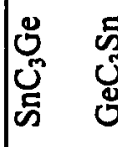 & 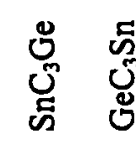 & 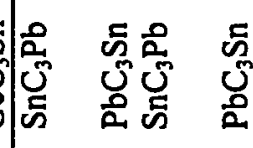 & 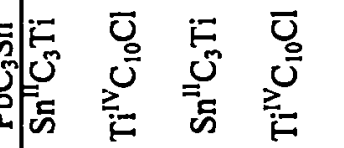 \\
\hline 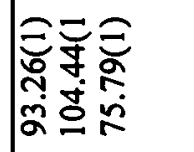 & & & & 夸 & 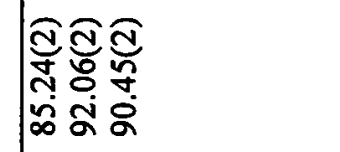 \\
\hline 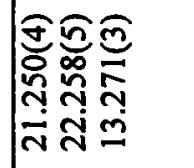 & & 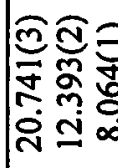 & & 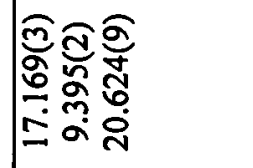 & 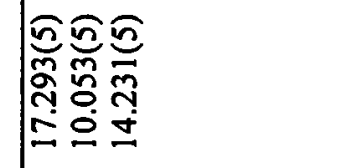 \\
\hline$=\bar{i} v$ & & 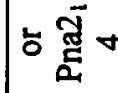 & & $a \frac{5}{\Sigma}$ & $=\bar{a} \sigma$ \\
\hline 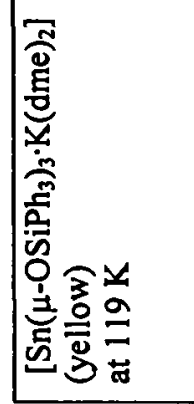 & & 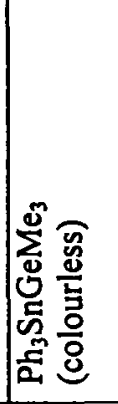 & & 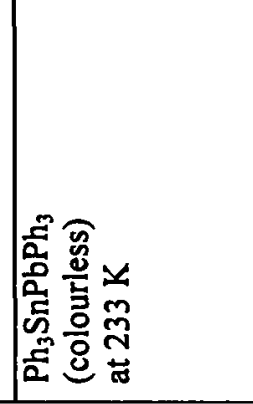 & 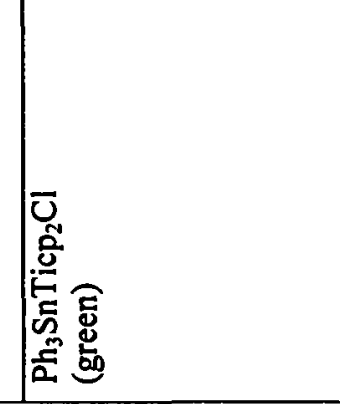 \\
\hline
\end{tabular}




\begin{tabular}{|c|c|c|c|c|c|c|c|c|c|c|}
\hline घ̈ & \multicolumn{3}{|l|}{$=$} & $\infty$ & \multicolumn{3}{|l|}{$a$} & \multicolumn{3}{|l|}{ సి } \\
\hline$\sum_{1}^{2}=$ & \multicolumn{3}{|c|}{ 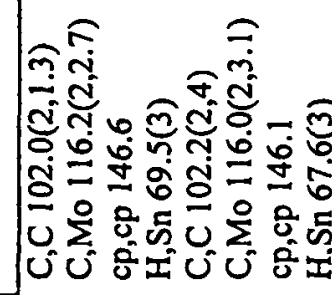 } & 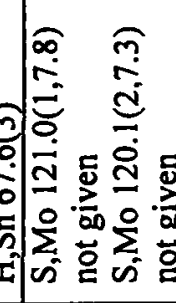 & \multicolumn{3}{|c|}{ 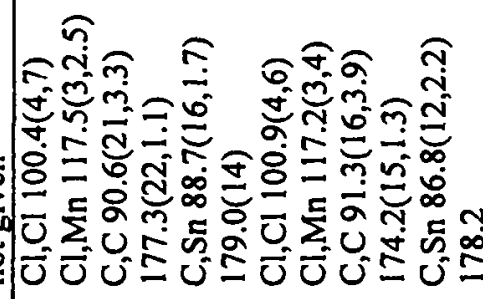 } & \multicolumn{3}{|c|}{ 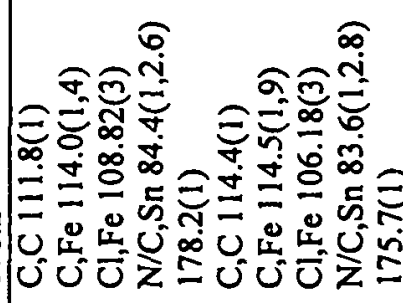 } \\
\hline$\sum_{1}=$ & 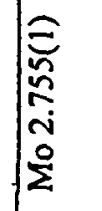 & 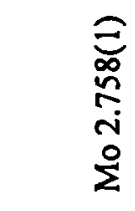 & & 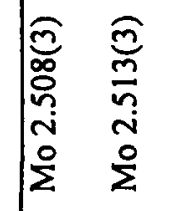 & 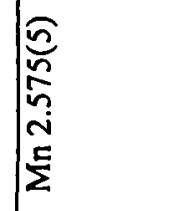 & 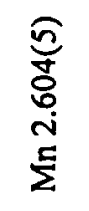 & & 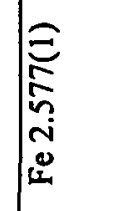 & 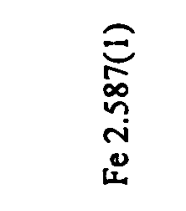 & \\
\hline$\frac{2}{1} \bar{x}$ & 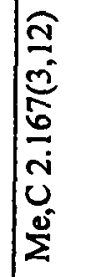 & 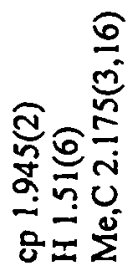 & 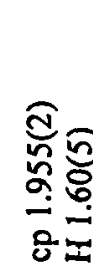 & 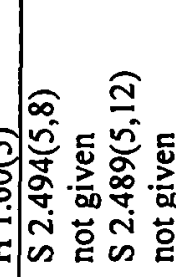 & 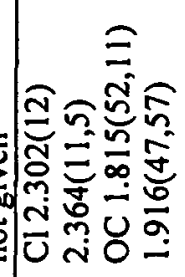 & 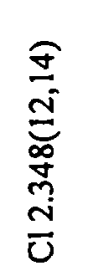 & 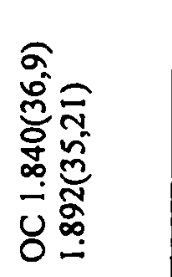 & 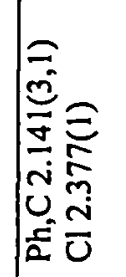 & 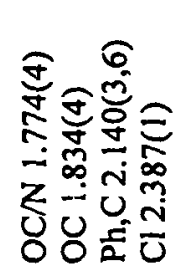 & 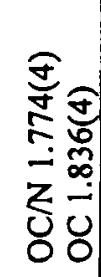 \\
\hline 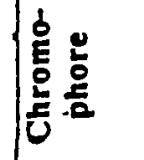 & 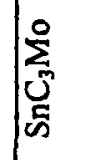 & 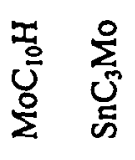 & 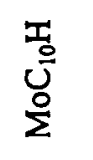 & 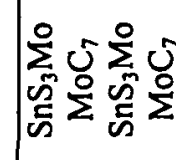 & 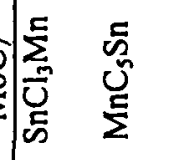 & 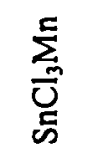 & 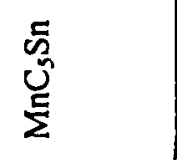 & 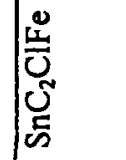 & 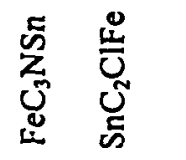 & 点 \\
\hline E: & & 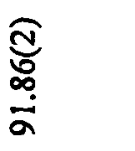 & & 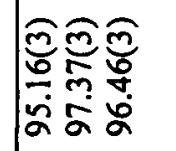 & 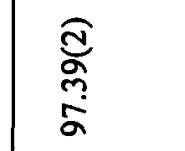 & & & 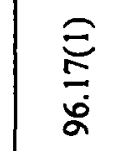 & & \\
\hline$\leq \frac{\pi}{0}$ & 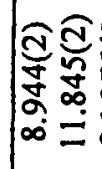 & & & 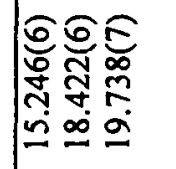 & 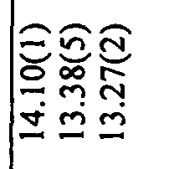 & & & 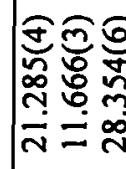 & & \\
\hline 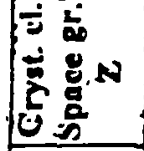 & E & & & $=\bar{a}$ & $E \stackrel{0}{\mathbb{N}^{\circ}}$ & & & E & & \\
\hline 룽 & 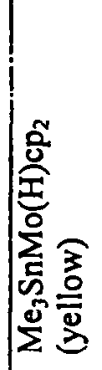 & & & 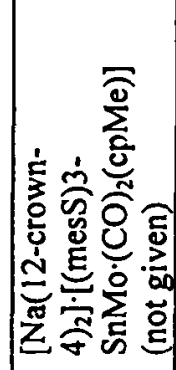 & 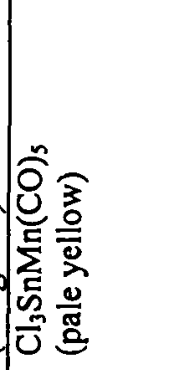 & & & 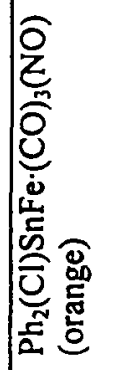 & & \\
\hline
\end{tabular}




\begin{tabular}{|c|c|c|c|c|c|c|c|c|c|}
\hline \multicolumn{3}{|l|}{$\bar{N}$} & \multicolumn{3}{|l|}{ 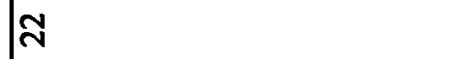 } & \multicolumn{4}{|l|}{$\tilde{\sim}$} \\
\hline \multicolumn{3}{|c|}{ 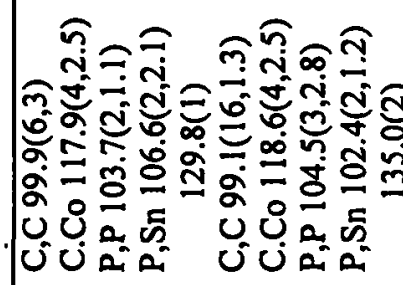 } & \multicolumn{3}{|c|}{ 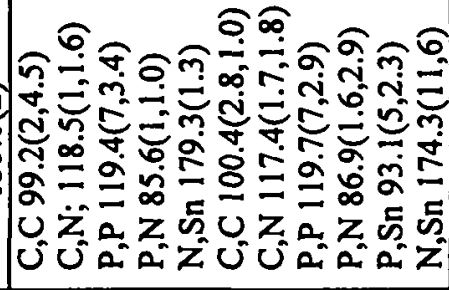 } & \multicolumn{4}{|c|}{ 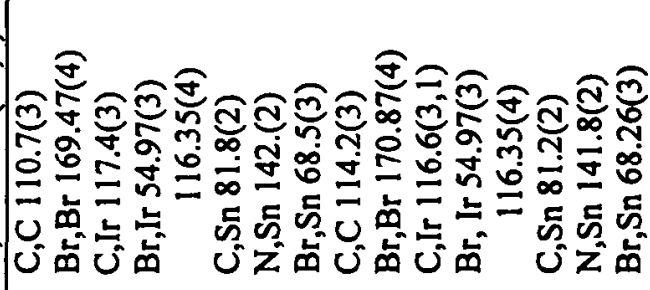 } \\
\hline 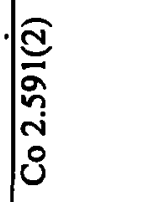 & $\begin{array}{l}\widehat{0} \\
o \\
\sigma \\
\tilde{\sigma} \\
0 \\
0\end{array}$ & & 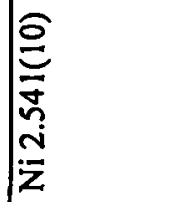 & 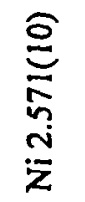 & & 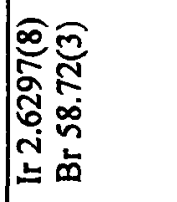 & & 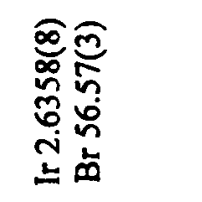 & \\
\hline 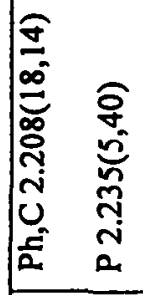 & 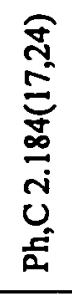 & 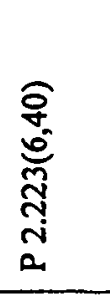 & 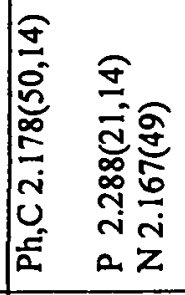 & 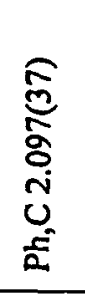 & 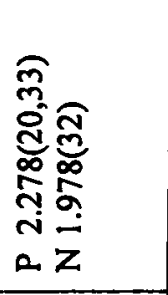 & 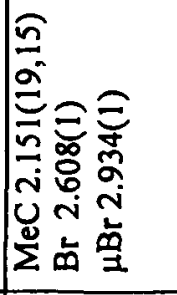 & 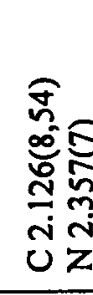 & 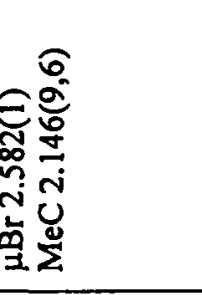 & 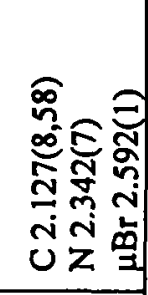 \\
\hline \multirow[t]{2}{*}{ 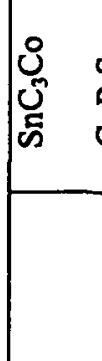 } & 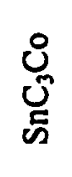 & $\begin{array}{l}\bar{p} \\
\text { on } \\
0 \\
0\end{array}$ & 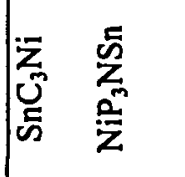 & & 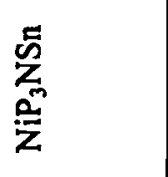 & 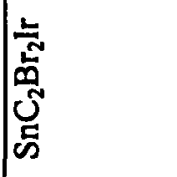 & 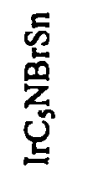 & 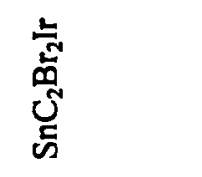 & 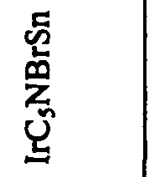 \\
\hline & & & \multicolumn{3}{|l|}{ 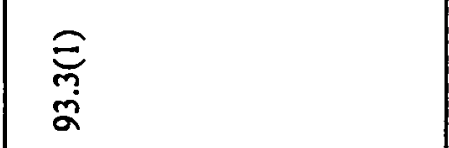 } & \multicolumn{4}{|l|}{ 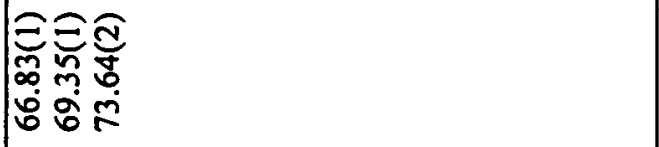 } \\
\hline \multicolumn{3}{|l|}{ 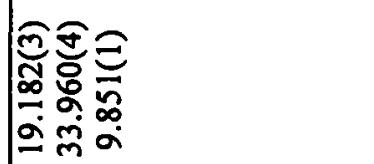 } & \multicolumn{3}{|l|}{ 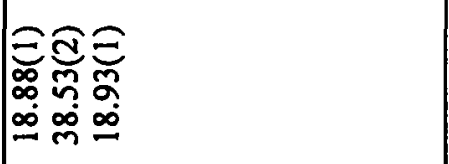 } & \multicolumn{4}{|l|}{ 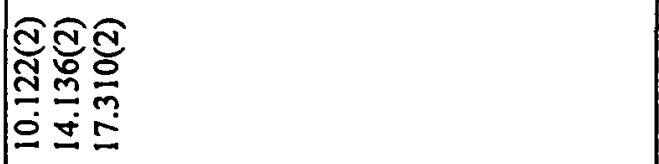 } \\
\hline \multicolumn{3}{|l|}{ ¿ } & \multicolumn{3}{|l|}{$E \stackrel{0}{\stackrel{0}{\Sigma}}$} & \multicolumn{4}{|l|}{$E \bar{a} \sigma$} \\
\hline \multicolumn{3}{|l|}{ 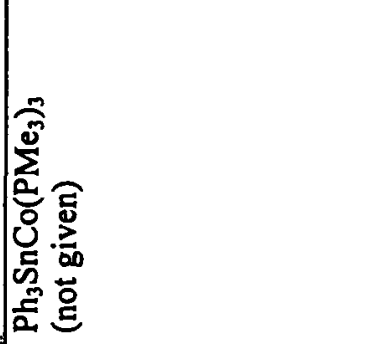 } & \multicolumn{3}{|l|}{ 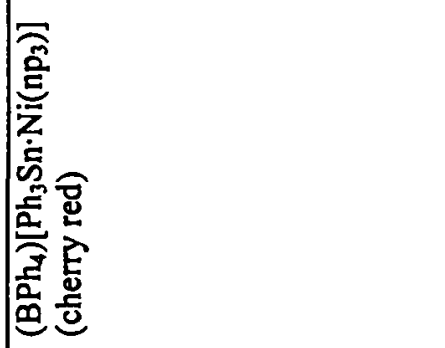 } & \multicolumn{4}{|l|}{ 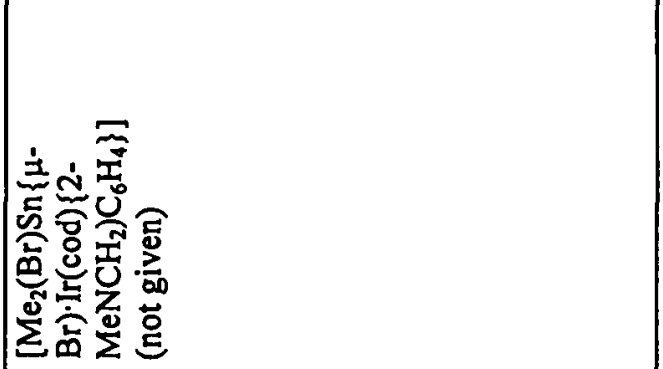 } \\
\hline
\end{tabular}




\begin{tabular}{|c|c|c|c|c|c|c|}
\hline \multirow{2}{*}{ 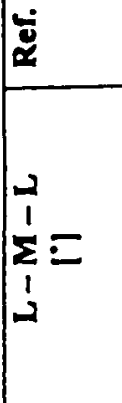 } & \multicolumn{3}{|l|}{ I } & \multicolumn{2}{|l|}{$\approx$} & \multirow{2}{*}{ 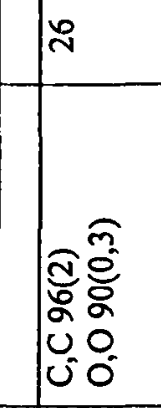 } \\
\hline & \multicolumn{3}{|c|}{ 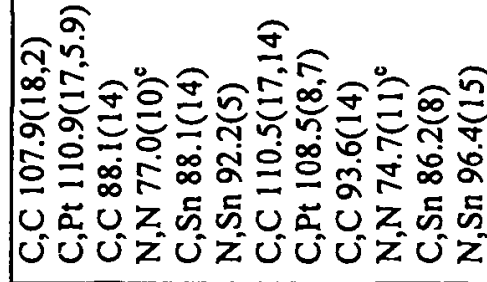 } & \multicolumn{2}{|c|}{ 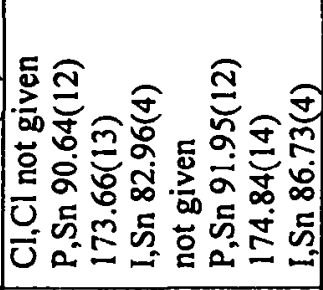 } & \\
\hline$\sum_{\substack{1 \\
\infty}} \bar{z}$ & 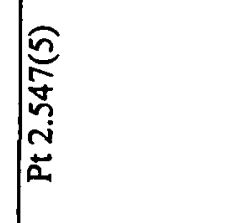 & & 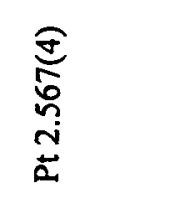 & 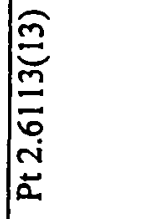 & 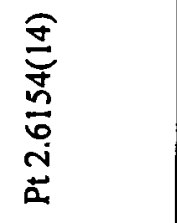 & \\
\hline$\frac{1}{2}=$ & 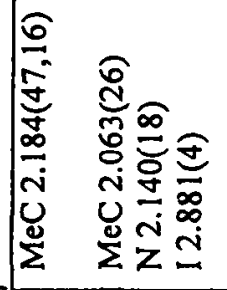 & & 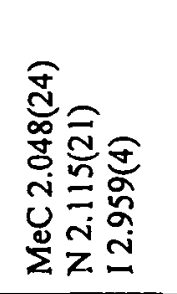 & 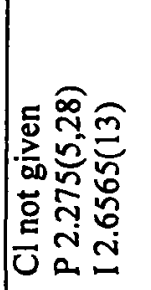 & 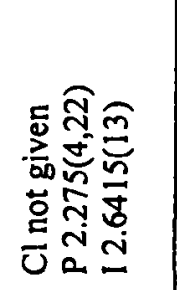 & 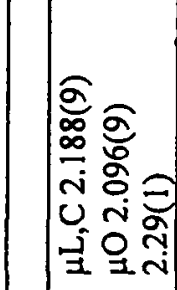 \\
\hline 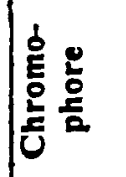 & 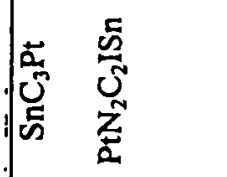 & & 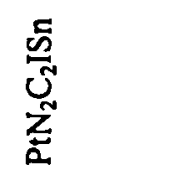 & 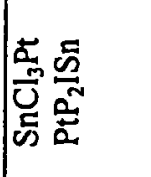 & 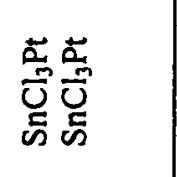 & 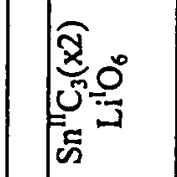 \\
\hline$=$ & 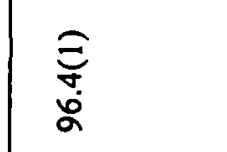 & & & 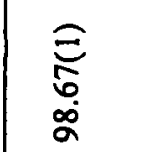 & & 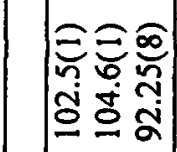 \\
\hline 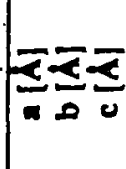 & 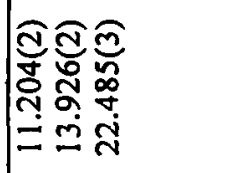 & & & 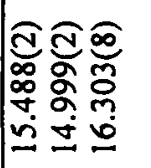 & & 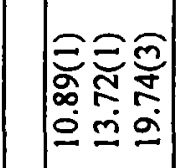 \\
\hline 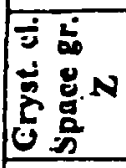 & $E \frac{E}{\Sigma} N$ & & & $E \bar{\Sigma}+$ & & $=\bar{a} N$ \\
\hline 施 & 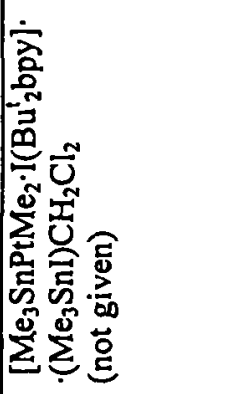 & & & 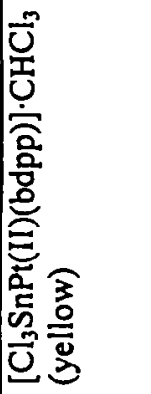 & & 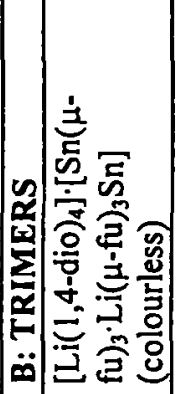 \\
\hline
\end{tabular}




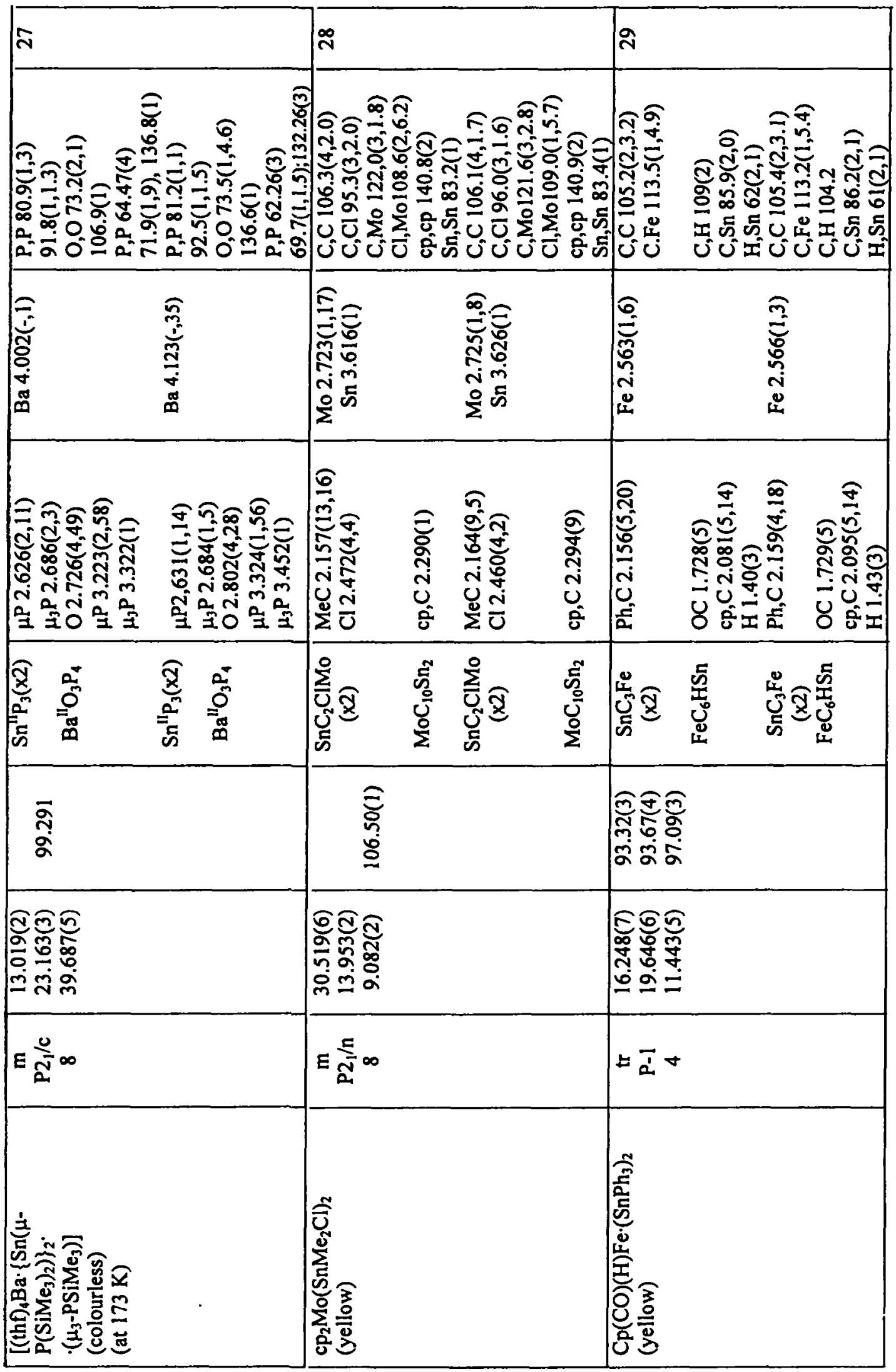




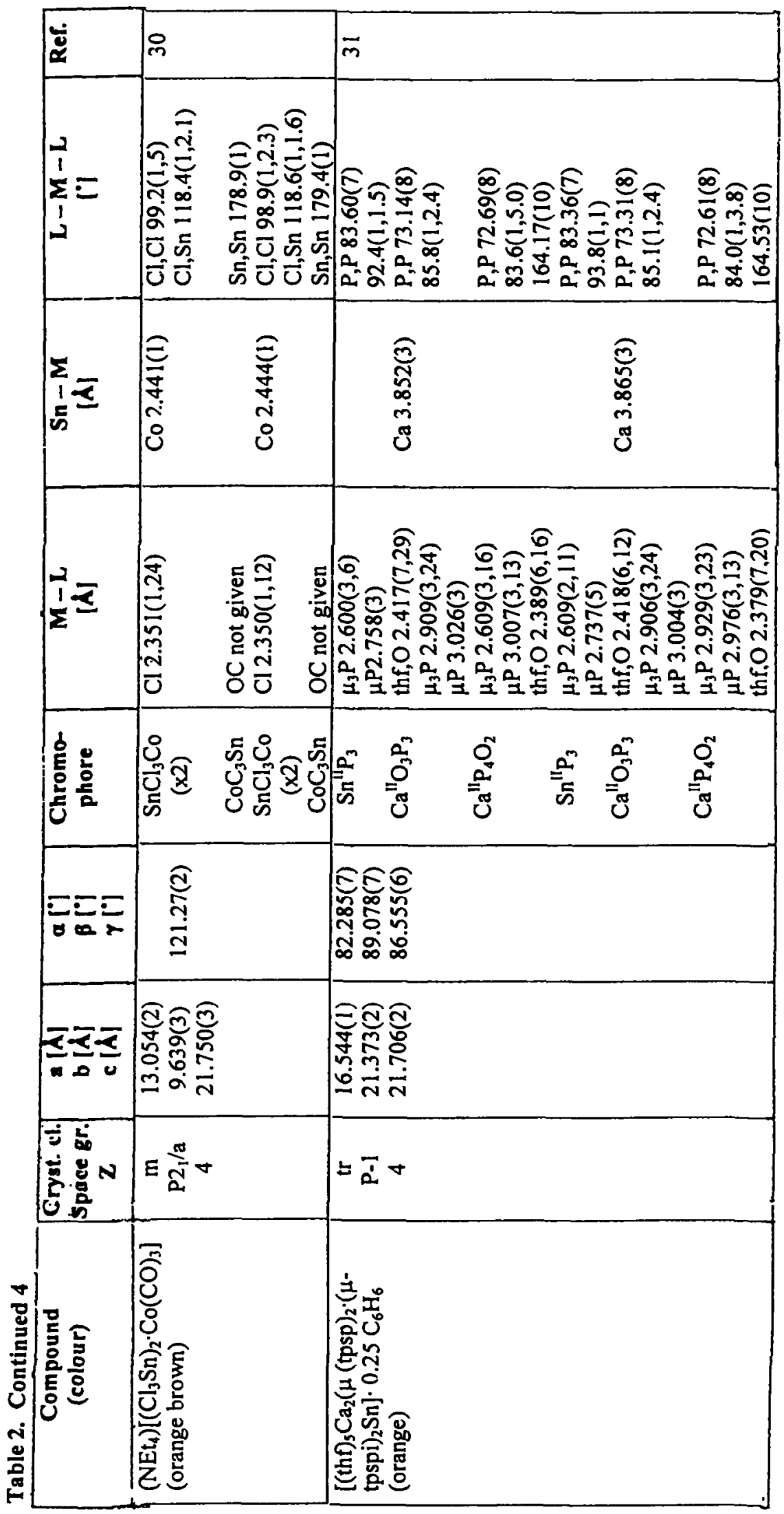




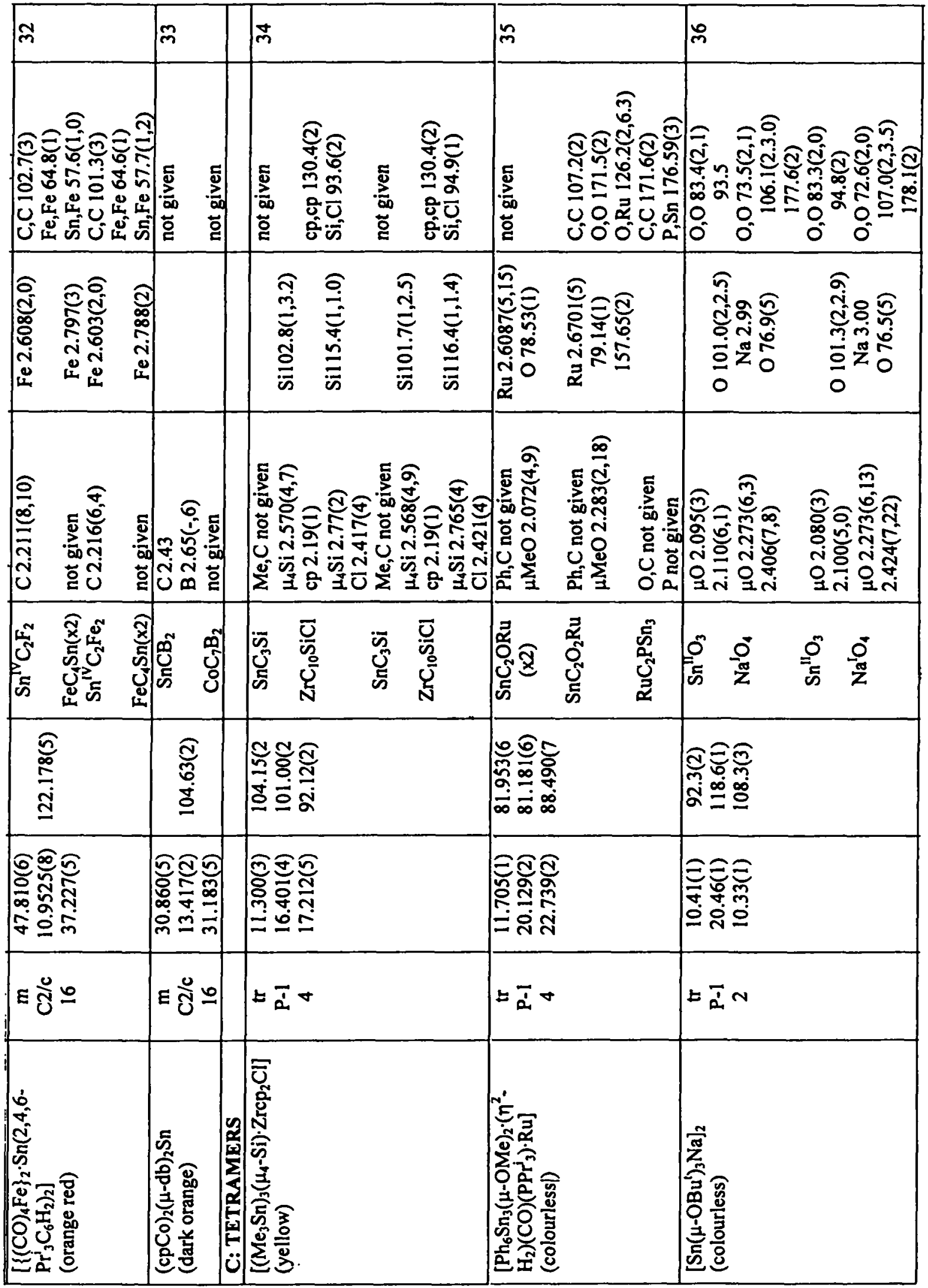




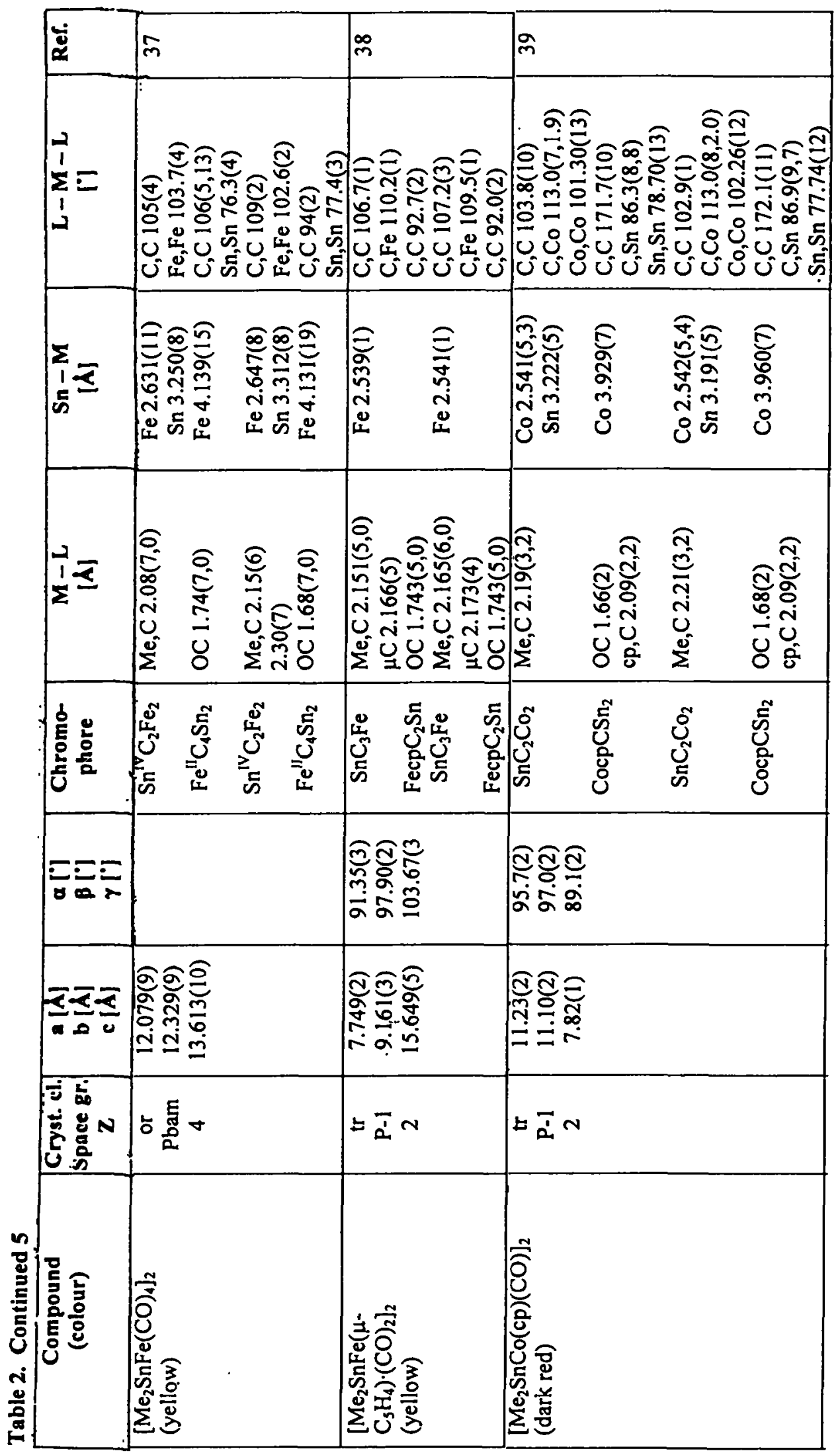




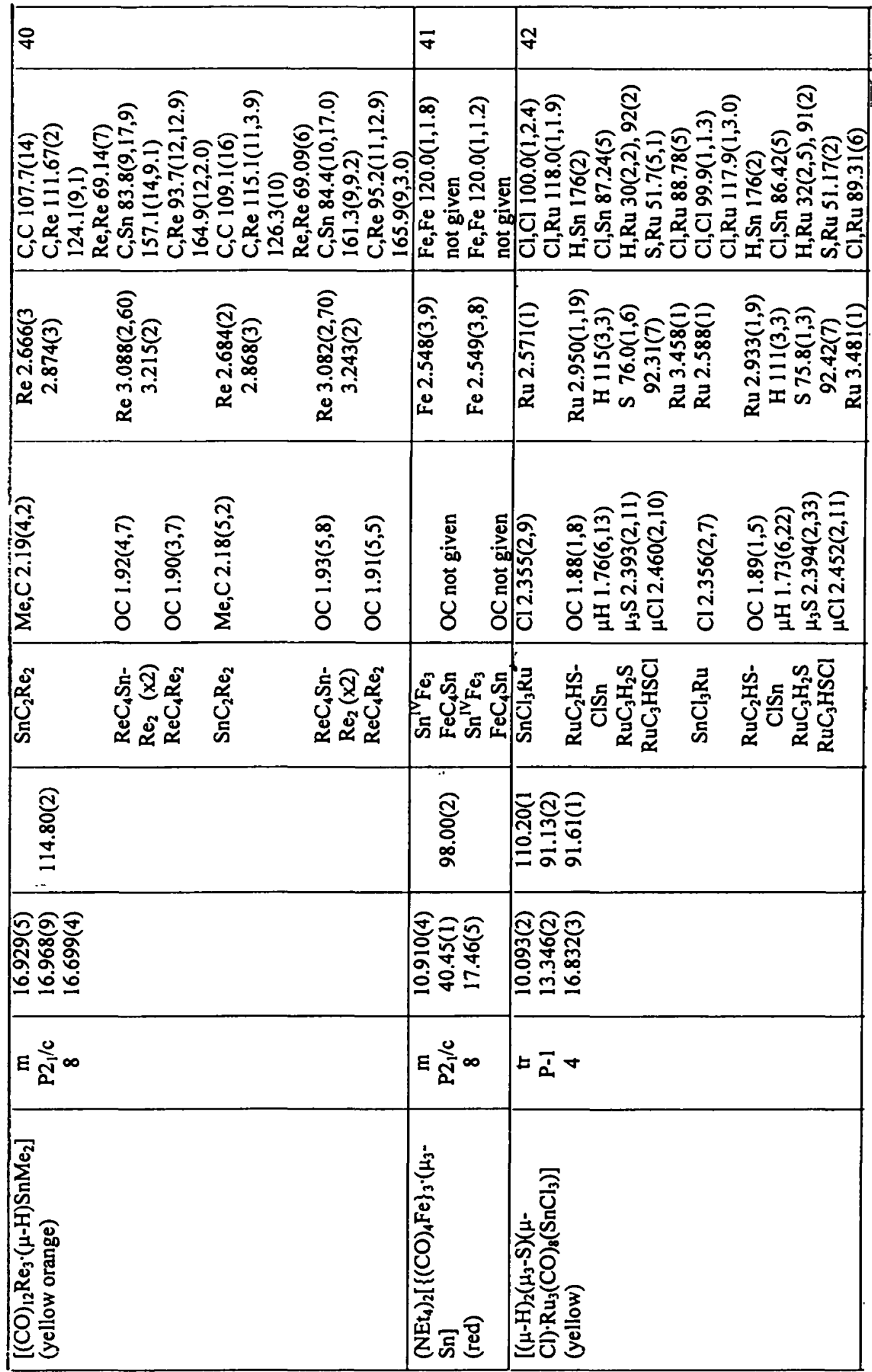




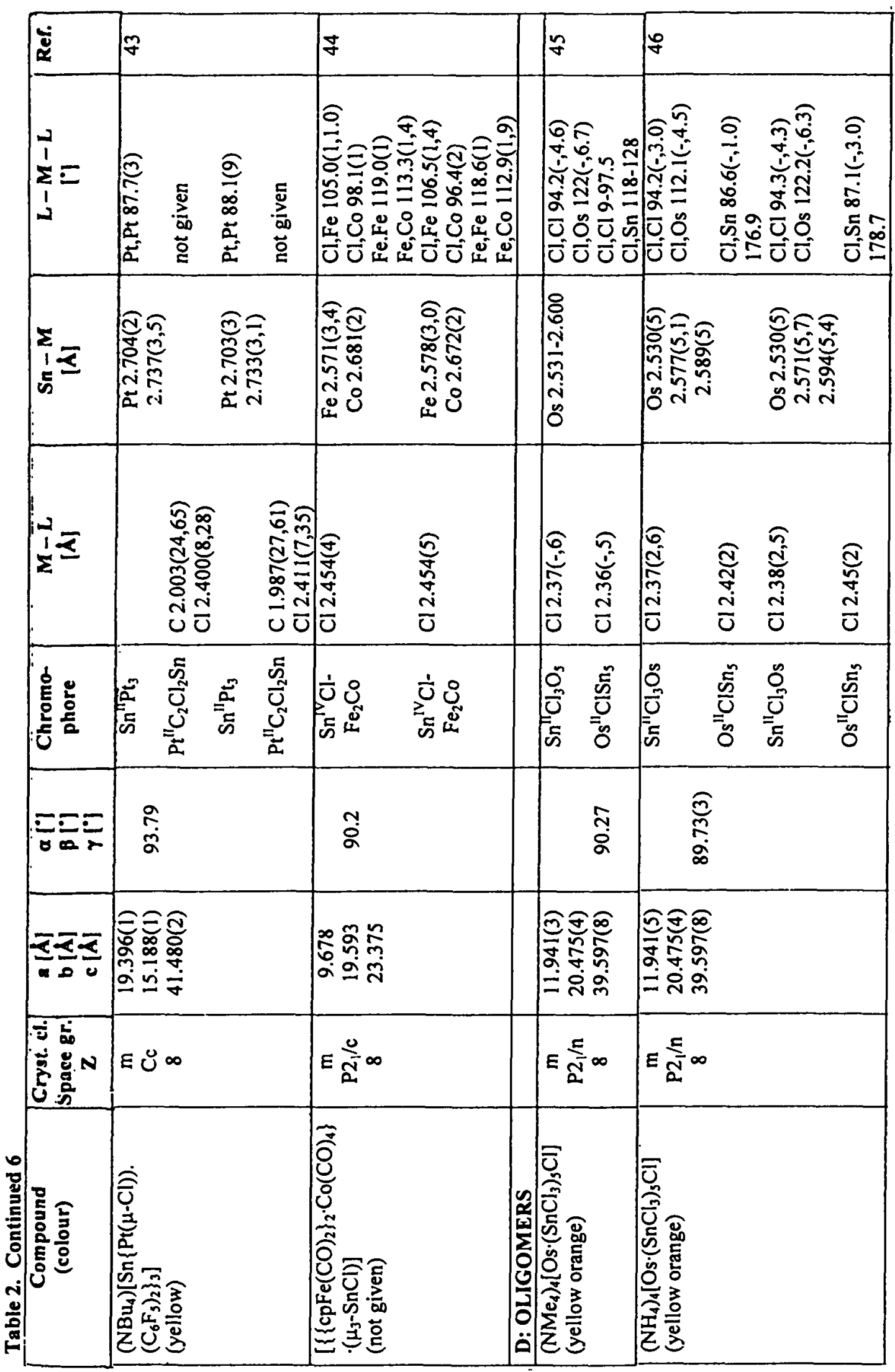




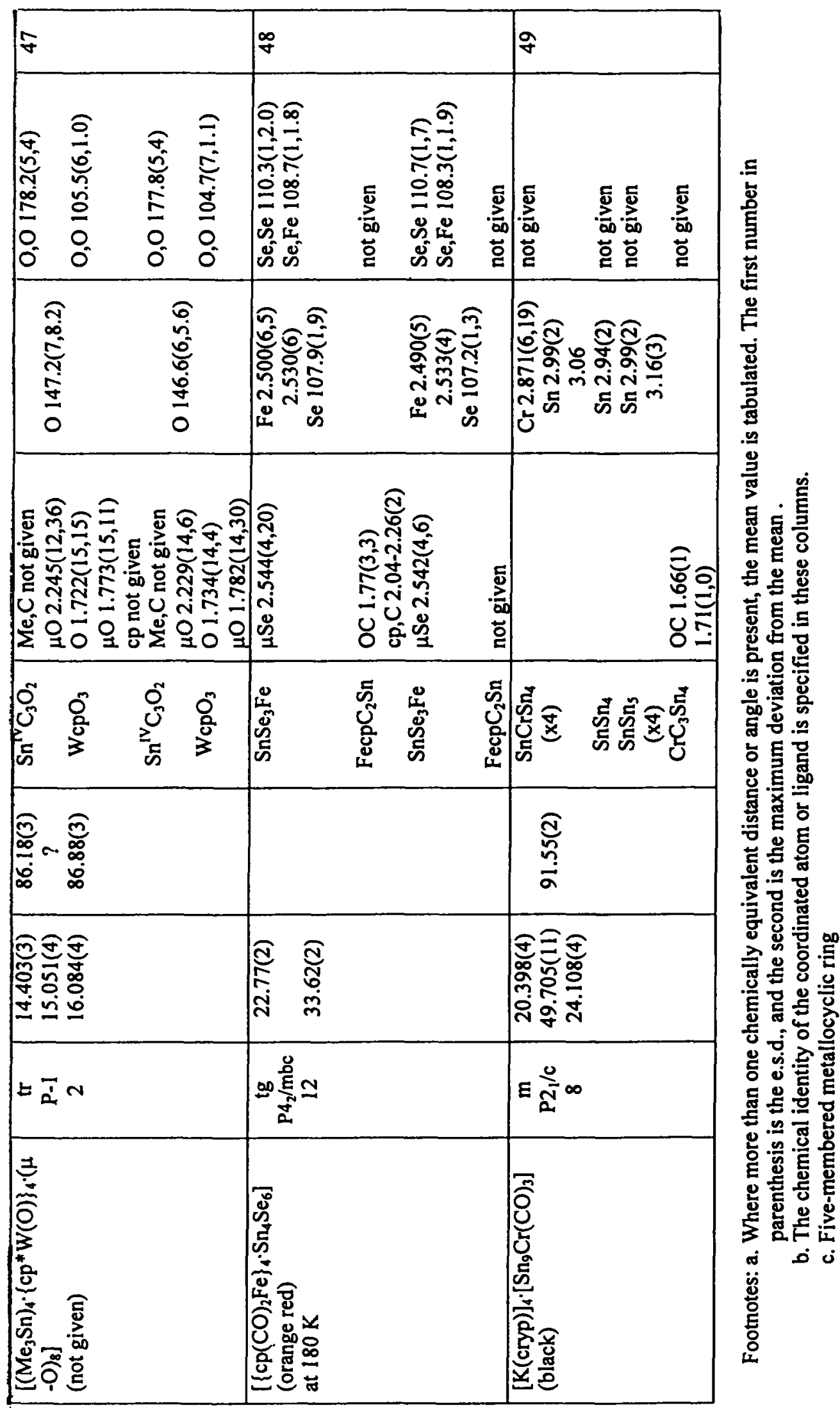


In pale yellow SnMn $/ 19 /$, orange $\mathrm{SnFe} / 20 /$, SnCo $/ 21 /$ and cherry red $\mathrm{SnNi} / 22 /$ derivatives (each contains two independent molecules), two non - equivalent moieties (Table 2A) are held together by a direct $\mathrm{Sn}-\mathrm{M}$ bonds with the lengths of 2.575(5) and 2.604(5) $\AA / 19 /, 2.577(1)$ and $2.587(1) / 20 /, 2.591(2)$ and $2.598(2) \AA / 21 /, 2.541(10)$ and $2.571 \AA / 22 /$. Each Sn atom is tetrahedrally coordinated $\left(\mathrm{SnCl}_{3} \mathrm{Mn}_{2}, \mathrm{SnC}_{2} \mathrm{ClFe}\right.$, $\mathrm{SnC}_{3} \mathrm{Co}$ and $\mathrm{SnC}_{3} \mathrm{Ni}$ ), with a different degree of distortion (Table $2 \mathrm{~A}$ ).

Structure of SnIr derivative $/ 23 /$ is shown in Fig. 3. As can be seen $\mathrm{Me}_{2}(\mathrm{Br}) \mathrm{Sn}$ and Ir (cod)\{2$\left.\left(\mathrm{MeNCH}_{2}\right) \mathrm{C}_{6} \mathrm{H}_{4}\right\}^{-}$units are held together by a bromine atom bridge as well as a direct $\mathrm{Sn}$ - Ir bond. The Sn Ir bonds are 2.6297(8) $\AA$ (molecule 1) and 2.6358(8) $\AA$ (molecule 2). The $\mathrm{Sn}-\mathrm{Br}-\mathrm{Ir}$ bridge angles are $58.72(3)$ and $56.57(3)^{\circ}$, respectively. Each tin atom has a trigonal - bipyramidal arrangement $\left(\mathrm{SnC}_{2} \mathrm{Br}_{2} \mathrm{Ir}\right)$, the $\mathrm{Br}$ atoms occupying apical positions $\left(\mathrm{Br}-\mathrm{Sn}-\mathrm{Br}\right.$ angle of $169.47(4)$ and $\left.170.87(4)^{\circ}\right)$.

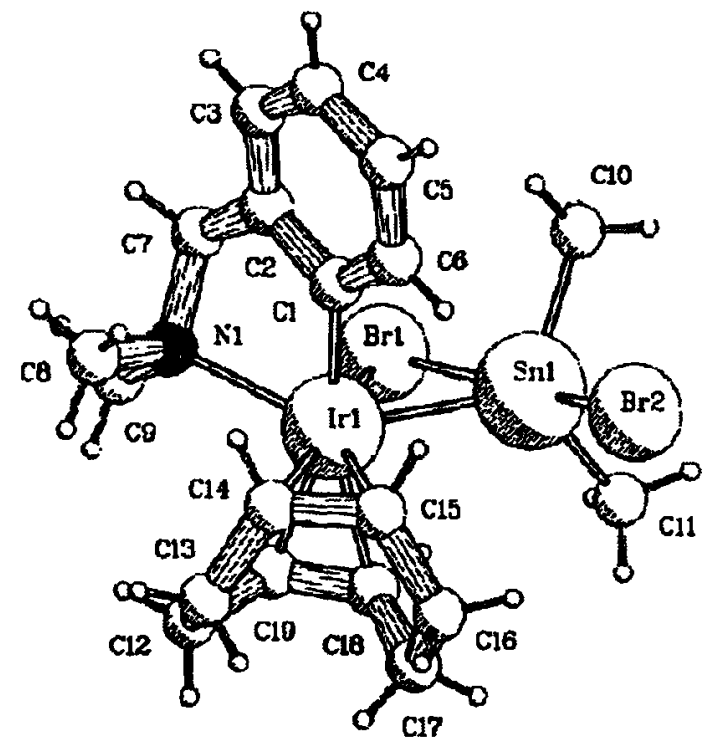

Fig. 3: Structure of $\left[\mathrm{Me}_{2}(\mathrm{Br}) \mathrm{Sn}(\mu-\mathrm{Br}) \operatorname{Ir}(\operatorname{cod})\left\{2\left(\mathrm{meNCH}_{2}\right) \mathrm{c}_{6} \mathrm{H}_{4}\right\}\right] \quad / 24 /$

In yellow SnPt derivative $/ 25 /$, only a direct $\mathrm{Sn}-\mathrm{Pt}$ bond holds together two non - equivalent $\left(\mathrm{Cl}_{3} \mathrm{Sn}\right.$ and Pt(bdpp)I) moieties with the length of 2.6113(13) $\AA$ (molecule 1) and 2.6154(14) $\AA$ (molecule 2).

\subsubsection{Trimers}

There are eight trimers $\left(\mathrm{Sn}_{2} \mathrm{M}(\mathrm{x} 5), \mathrm{SnM}_{2}(\mathrm{x} 3)\right)$ which contain two crystallographically independent molecules and their structural parameters are gathered in Table 2B.

A colourless $\mathrm{Sn}_{2} \mathrm{Li}$ cluster $/ 26 /$ contains a [ $\left.\mathrm{Li}(1,4-\text { dioxane })_{4}\right]^{+}$cation and a $\left[\mathrm{Sn}(\mu \text { - furyl })_{3} \mathrm{Li}(\mu \text {-furyl })_{3} \mathrm{Sn}\right]^{-}$ anion. In the latter the central $\mathrm{Li}(\mathrm{I})$ atom has six furyl $\mathrm{O}$ atoms in an octahedral arrangement $\left(\mathrm{LiO}_{6}\right)$. Each satellite $\mathrm{Sn}$ (II) atom is bonded by three furyl carbon atoms in a typical pyramidal arrangement with C - Sn $\mathrm{C}$ angles of $96(2)^{\circ}$.

Barium(II) atom is the "central " atom in another colourless $\mathrm{Sn}_{2} \mathrm{Ba}$ derivative $/ 27 /$ and is linked to four $\mathrm{P}$ atoms of $\mathrm{P}\left(\mathrm{SiMe}_{3}\right)_{2}$ groups which also bridge to the two outer $\mathrm{Sn}(\mathrm{II})$ atoms. These are also coordinated by terminal $\mathrm{P}\left(\mathrm{SiMe}_{3}\right)_{2}$ groups to give a pyramidal $\mathrm{SnP}_{3}$ geometry. The mean $\mathrm{Sn}-\mathrm{Ba}$ separation of $4.002 \AA$ (molecule 1) and $4.123 \AA$ (molecule 2 ) rules out a direct bond. 
A yellow $\mathrm{Sn}_{2} \mathrm{Mo}$ derivative /28/ also consists of two crystallographically independent molecules differing mostly by degree of distortion. The Mo atom is $\pi$ - bonded to two cyclopentadienyl rings and through $\sigma$ bonds to two Sn atoms (mean Sn - Mo bond distances of 2.723(1) Á (molecule 1) and 2.725(1) Á (molecule 2)). The $\mathrm{Sn}-\mathrm{Sn}$ distances of 3.616(1) and 3.626(1) $\AA$ indicate the absence of any direct bonding. Each tin atom is a tetrahedrally coordinated $\left(\mathrm{SnC}_{2} \mathrm{ClMo}\right)$.

Structure of another yellow $\mathrm{cp}(\mathrm{CO})(\mathrm{H}) \mathrm{Fe}\left(\mathrm{SnPh}_{3}\right)_{2}$ derivative $/ 29 / \mathrm{can}$ be described as a four legged piano stool with the $\mathrm{SnPh}_{3}$ groups occupying the mutually trans basal positions. The mean $\mathrm{Sn}-\mathrm{Fe}$ bond distances are 2.563(1) and 2.566(1) $\AA$, respectively.

Structure of orange brown $\mathrm{Sn}_{2} \mathrm{Co}$ derivative $130 /$ consists of well separated $\mathrm{NEt}_{4}$ cation and $\left[\left(\mathrm{Cl}_{3} \mathrm{Sn}\right)_{2} \mathrm{Co}(\mathrm{CO})_{3}\right]^{-}$anion. In the complex anion, the central $\mathrm{Co}(\mathrm{CO})_{3}$ unit is bound to two $\mathrm{SnCl}_{3}$ units with mean Co - Sn bond distances of 2.441(1) and 2.444(1) $\AA$. Each tin atom is tetrahedrally coordinated $\left(\mathrm{SnCl}_{3} \mathrm{Co}\right)$.

Structure of orange $\mathrm{SnCa}_{2}$ derivative $/ 31 /$ has a three-coordinated $\mathrm{Sn}(\mathrm{II})$ atom( $\left(\mathrm{SnP}_{3}\right)$ with six-coordinated $\mathrm{Ca}(\mathrm{II})$ atoms $\left(\mathrm{CaO}_{3} \mathrm{P}_{3}\right.$ and $\left.\mathrm{CaP}_{4} \mathrm{O}_{2}\right)$ Fig. 4. The mean separation of $3.852(3)$ and 3.865(3) $\AA$, between two $\mathrm{Ca}$ (II) atoms excludes a real metal - metal bond.

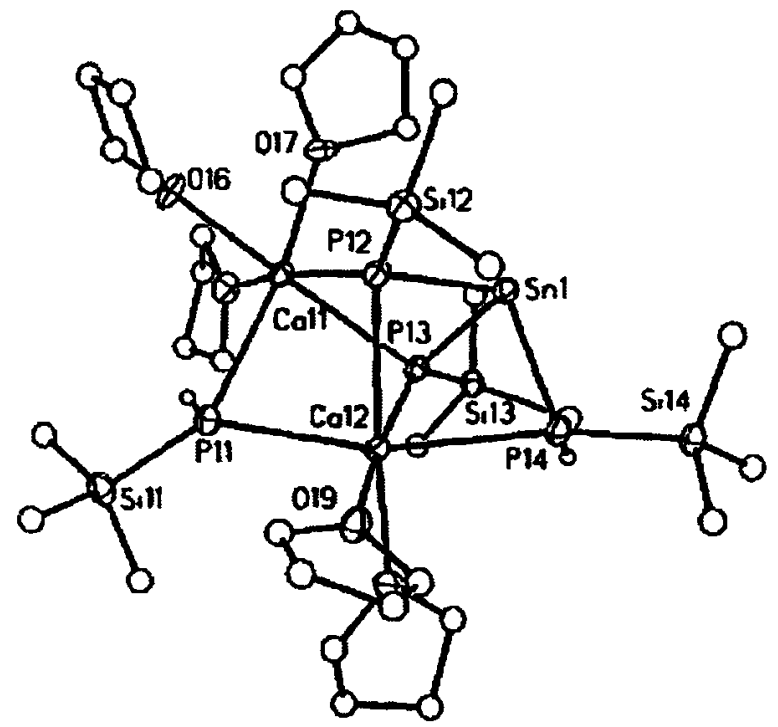

Fig. 4: Structure of $\left[(\text { thf })_{5} \mathrm{Ca}_{2}(\mathrm{tppp})_{2}(\mu \text {-tpspi })_{2} \mathrm{Sn}\right] / 31 /$

Structure of orange red $\left[\left\{(\mathrm{OC})_{4} \mathrm{Fe}\right\}_{2} \mathrm{Sn}\left(2,4,6-\mathrm{Pr}_{3}{ }_{3} \mathrm{C}_{6} \mathrm{H}_{2}\right)_{2}\right] / 32 /$ contains a metal triangle with mean $\mathrm{Sn}-\mathrm{Fe}$ bond distances $2.603(2)$ and 2.608(2) $\AA$ and $\mathrm{Fe}-\mathrm{Fe}$ bond distances of 2.788(2) and 2.797(3) $\AA$. The Fe - Sn $-\mathrm{Fe}$ angles are about $64.6(1)$ and $64.8(1)^{\circ}$, but the $\mathrm{Fe}-\mathrm{Fe}-\mathrm{Sn}$ angles are about $7^{\circ}$ smaller $\left(57.6^{\circ}\right.$ to $58.0(1)^{\circ}$, average $57.7^{\circ}$. 
Dark orange $\left[\{\mathrm{cpCo}(\mu-\mathrm{db})\}_{2} \mathrm{Sn}\right] / 33 /$ also contains two crystallographically independent molecules differing mostly by degree of distortion. Two virtually identical $\mathrm{cpCo}_{2}\left(\mathrm{C}_{2} \mathrm{~B}_{2} \mathrm{C}\right)$ sandwich units are bound to the central tin atom in a $\eta^{5}$ fashion via the bridging 1,3-biborolenyl ligands. The tetradecker complex exhibits a bent structure in which the $\mathrm{Sn}-\mathrm{Co}$ vectors form a $130^{\circ}$ angle.

\subsubsection{Tetramers}

There are eleven $\left(\mathrm{Sn}_{3} \mathrm{M}(\mathrm{x} 2), \mathrm{Sn}_{2} \mathrm{M}_{2}(\mathrm{x} 4), \mathrm{SnM}_{3}(\mathrm{x} 4), \mathrm{SnM}_{2} \mathrm{M}^{\prime}(\mathrm{x} 1)\right)$ tetramers and their structural parameters are gathered in Table $2 \mathrm{C}$.

A yellow derivative containing a $\mathrm{Sn}_{3}-\mathrm{Zr}$ cluster $/ 34 /$ has a $\mu$-Si atom serving as a bridge between three $\mathrm{Me}_{3} \mathrm{Si}$ moieties and one $\mathrm{Zncp}_{2} \mathrm{Cl}$ unit. The tin atoms are all in distorted tetrahedral environments $\left(\mathrm{SnC}_{3} \mathrm{Si}\right)$. The zirconocene cyclopentadienyl rings are eclipsed because of the incorporation of a $\mathrm{Cl}$ and a Si atom into the "sandwich" cavity. Two crystallographically independent $\mathrm{Sn}_{3} \mathrm{Zr}$ clusters are examples of distortion isomerism.

The structure of an orange $\mathrm{Sn}_{3} \mathrm{Ru}$ cluster is shown in Fig. 5, where it can be seen that the $(\mathrm{Ph})_{2} \mathrm{SnO}(\mathrm{Me}) \mathrm{Sn}\left(\mathrm{Ph}_{2}\right) \cdot \mathrm{O}(\mathrm{Me}) \mathrm{Sn}(\mathrm{Ph})_{2}$ occupies three coordination sites in a mer orientation around $\mathrm{Ru}$. The terminal tin atoms are both tetrahedral $\left(\mathrm{SnC}_{2} \mathrm{ORu}\right)$ and the central tin atom is in a distorted trigonal bipyramidal environment $\left(\mathrm{SnC}_{2} \mathrm{O}_{2} \mathrm{Ru}\right)$ with $\mathrm{O}$ atoms at apical positions $\left(\mathrm{O}-\mathrm{Sn}-\mathrm{O}^{\circ}=171.1^{\circ}\right)$. The $\mathrm{Sn}(2)$ $\mathrm{Ru}$ distance of 2.6701(5) $\AA$ is about $0.06 \AA$ longer than the remaining two $\mathrm{Sn}-\mathrm{Ru}$ distances (2.6072(5) and $2.6102(5)$ Á.

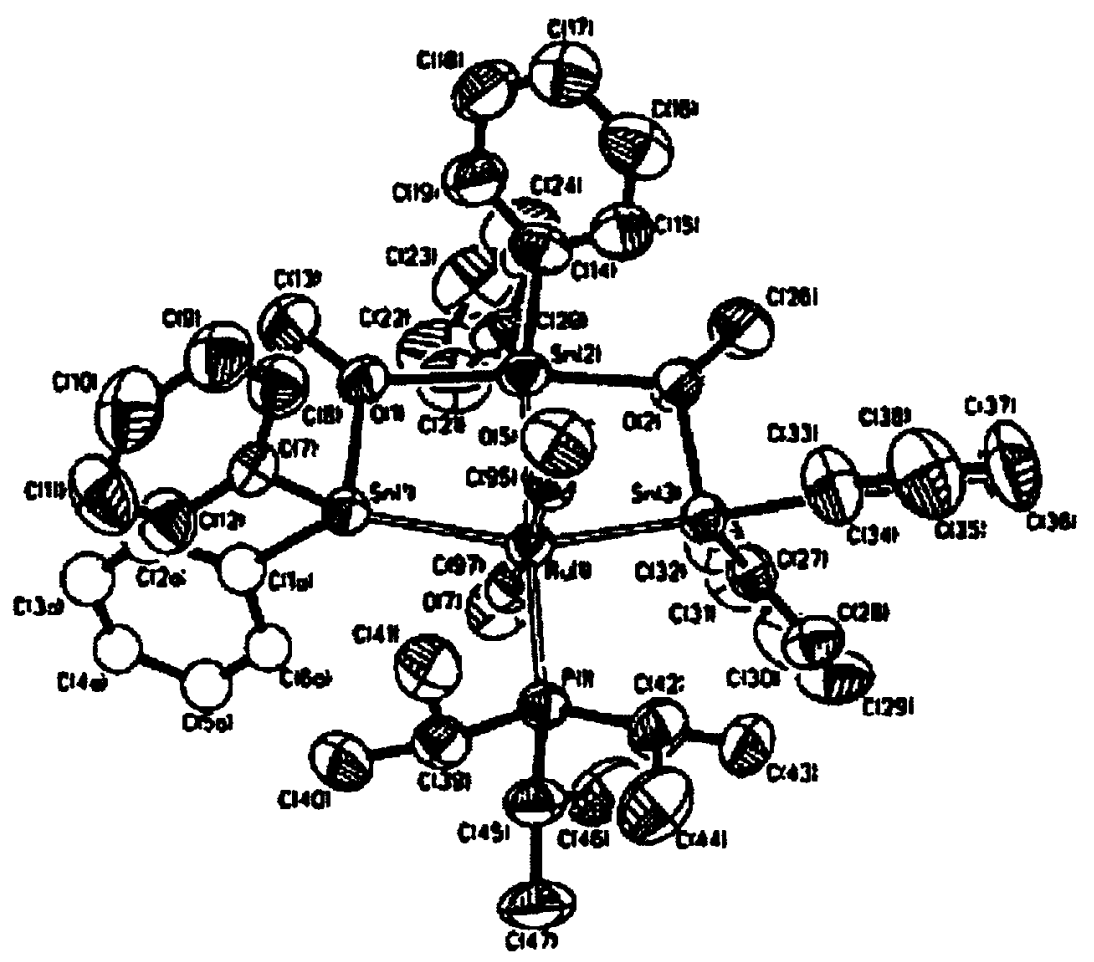

Fig. 5: Structure of $\left[\mathrm{Ph}_{6} \mathrm{Sn}_{3}(\mu-\mathrm{OMe})_{2}\left(\eta^{2}-\mathrm{H}_{2}\right)(\mathrm{CO})\left(\mathrm{PPr}_{3}{ }_{3}\right) \mathrm{Ru}\right] \quad / 35 /$ 
There are four clusters containing two pairs of metal atons, two tin atoms and two of the same heterometal atoms. A colourless $\left.\left[\mathrm{Sn}(\mu-\mathrm{OBu})_{3}\right)_{3} \mathrm{Na}\right]_{2}$ cluster $/ 36 /$ contains a $\mathrm{Sn}_{2} \mathrm{O}_{6} \mathrm{Na}_{2}$ cage which is constructed from two seminorcubane $\mathrm{Sn}_{2} \mathrm{Na}_{2} \mathrm{O}_{3}$ units sharing a $\mathrm{Na}_{2} \mathrm{O}_{2}$ four membered ring. The $\mathrm{Na}-\mathrm{Na}$ distance is $2.99 \AA$ (molecule 1 ) and $3.00 \AA$ (molecule 2). Each Sn(II) atom has a trigonal planar environment $\left(\mathrm{SnO}_{3}\right)$ with the sum of $\mathrm{Sn}-\mathrm{O}$ bond distances of $6.280 \AA$ in one and $6.315 \AA$ in the other molecule.

There are two yellow $\mathrm{Sn}_{2} \mathrm{Fe}_{2}$ clusters $/ 37,38 /$. The former $/ 37 /$ is characterized by a central lozenge shaped four-membered $\mathrm{Sn}_{2} \mathrm{Fe}_{2}$ ring with $\mathrm{Sn}-\mathrm{Fe}$ and $\mathrm{Sn}-\mathrm{Sn}$ distances of 2.631(11) and 3.250(8) $A$ in molecule 1 and 2.647(8) and 3.312(8) $\AA$ in molecule 2. The mean $\mathrm{Sn}-\mathrm{Fe}-\mathrm{Sn}$ and $\mathrm{Fe}-\mathrm{Sn}-\mathrm{Fe}$ bond angles are 77 and $103^{\circ}$, respectively. The structure of the other yellow $\mathrm{Sn}_{2} \mathrm{Fe}_{2}$ cluster $/ 38 /$ consists of pairs $\mathrm{Me}_{2} \mathrm{Sn}$ and $\mathrm{Fe}\left(\mu-\mathrm{C}_{5} \mathrm{H}_{4}\right)(\mathrm{CO})_{2}$, in which the $\mathrm{Sn}-\mathrm{Fe}$ bond distances are 2.539(1) $\AA$ (molecule 1) and 2.541(1) $\AA$ (molecule 2).

A dark red $\left[\mathrm{Me}_{2} \mathrm{SnCo}(\mathrm{cp})(\mathrm{CO})\right]_{2} / 39 /$ also contains two crystallographically independent molecules in the unit cell, each located astride a centre of inversion, but with no significant differences in molecular geometry. Tin and cobalt atoms alternate around the four-membered planar ring with angles at $\mathrm{Co}$ of $78^{\circ}$ and at $\mathrm{Sn}$ of $102^{\circ}$. The mean $\mathrm{Sn}-\mathrm{Co}, \mathrm{Sn}-\mathrm{Sn}$ and Co - Co distances are 2.541(5), 3.222(5) and 3.929(7) $\mathcal{A}$ in molecule $\mathrm{I}$ and 2.542(5), 3.1941(5) and 3.960(7) $\AA$ in molecule 2 . This indicates that the $\mathrm{Sn}-\mathrm{Co}$ is a real bond while the other two are not. Both tin atoms are in tetrahedral environments $\left(\mathrm{SnC}_{2} \mathrm{Co}_{2}\right)(\mathrm{Table} 2 \mathrm{C})$.

In a yellow orange $\left[(\mathrm{CO})_{12} \mathrm{Re}_{3}(\mu-\mathrm{H}) \mathrm{SnMe}_{2}\right]$ cluster $/ 40 /$, the asymmetric unit contains two similar but crystallographically independent molecules, examples of distortion isomerism. In each the three $\operatorname{Re}$ atoms define an approximate equilateral triangle, with the $\mathrm{Sn}$ atom bridging one edge essentially in the plane of the triangle. The $\mathrm{Sn}-\mathrm{Re}$ bond distances are 2.666(3) and 2.874(3) $\AA$ in one molecule, 2.684(2) and 2.868(3) $\AA$ in the other. The $\mathrm{Me}_{2} \mathrm{Sn}$ bridged $\mathrm{Re}-\mathrm{Re}$ distances are 3.148(2) and 3.152(2) $\AA$, respectively, and the mean value of the other $\operatorname{Re}$ - Re distances is shorter, at $3.08 \AA$. Each thenium atom has four terminal carbonyl groups.

Structure of red $\mathrm{SnFe}_{3}$ cluster $/ 41 /$ contains well separated $\mathrm{NEt}_{4}{ }^{+}$cations and $\left.\left[\{\mathrm{CO})_{4} \mathrm{Fe}\right\}_{3}\left(\mu_{3}-\mathrm{Sn}\right)\right]^{2-}$ anion. In the cluster anion, the four metal atoms form a core with mean $\mathrm{Sn}-\mathrm{Fe}$ bond distances 2.548(3) $\AA$ in one molecule and $2.549(3)$ in the other. The $\mathrm{Fe}-\mathrm{Sn}-\mathrm{Fe}$ angles are all very close to $120^{\circ}$ (maximum deviation $1.8^{\circ}$ (molecule 1 ) and $1.2^{\circ}$ (molecule 2)).

A yellow $\left[(\mu-\mathrm{H})_{2}\left(\mu_{3}-\mathrm{S}\right)(\mu-\mathrm{Cl}) \mathrm{Ru}_{3}(\mathrm{CO})_{8}\left(\mathrm{SnCl}_{3}\right)\right]$ cluster /42/ contains two independent molecules of essentially identical structure. The three $\mathrm{Ru}$ atoms are in a triangular cluster with only two metal - metal bonds which also have a bridging hydride ligand. A sulphide ligand bridges all three metal atoms together. Two of the Ru atoms carry three terminal carbonyl groups, while the third has two carbonyls and a trichlorostannyl group which lies on the sulphur bridged side of the Ru plane. The $\mathrm{Sn}-\mathrm{Ru}$ distance is $2.571(1) \AA$ in one molecule and $2.588(1) \AA$ in the other. The ruthenium atoms differ in stereochemistry $\left(\mathrm{RuC}_{2} \mathrm{HSClSn}, \mathrm{RuC}_{3} \mathrm{H}_{2} \mathrm{~S}\right.$ and $\left.\mathrm{RuC}_{3} \mathrm{HSCl}\right)$ and the tin atom has a distorted tetrahedral arrangement $\left(\mathrm{SnCl}_{3} \mathrm{Ru}\right)$. 
A yellow $\mathrm{SnPt}_{3}$ cluster $/ 43 /$ contains two crystallographically independent $\left[\operatorname{Sn}\left\{\operatorname{Pt}(\mu-\mathrm{Cl})\left(\mathrm{C}_{6} \mathrm{~F}_{5}\right)_{2}\right\}_{3}\right]^{-}$anions. The inner platinum moiety can be described as a puckered $\mathrm{Pt}_{3} \mathrm{Cl}_{3}$ ring, in which the $\mathrm{Pt}(\mathrm{II})$ atoms are interconnected by the $\mathrm{Cl}$ atoms and which acts as a tridentate donor - acceptor ligand to the $\mathrm{Sn}$ (II) atom. Each $\mathrm{Pt}(\mathrm{II})$ atom has a square - pyramidal coordination $\left(\mathrm{PtC}_{2} \mathrm{Cl}_{2} \mathrm{Sn}\right)$ with the $\mathrm{Sn}$ atom at the apex of each. The mean Sn - Pt bond distances are $2.723 \AA$ in one molecule and $2.726 \AA$ in the other.

In $\left.\left.[\{\mathrm{cpFe}\} \mathrm{CO})_{2}\right\}_{2} \mathrm{Co}(\mathrm{CO})_{4}\left(\mu_{3}-\mathrm{SnCl}\right)\right]$ cluster $/ 44 /$ a central $\mathrm{Sn}(\mathrm{IV})$ atom has a tetrahedral arrangement $\left(\mathrm{SnClFe}_{2} \mathrm{Co}\right)$ with the $\mathrm{Sn}-\mathrm{Fe}$ and $\mathrm{Sn}-\mathrm{Co}$ bond distances of 2.571 (mean) and 2.681(2) $\AA$ in one molecule and 2.578 (mean) and 2.672(2) $\AA$ in the other (Table 2C).

\subsubsection{Oligomers}

There are five $\left(\mathrm{Sn}_{5} \mathrm{Os}(\mathrm{x} 2), \mathrm{Sn}_{4} \mathrm{M}_{4}(\mathrm{x} 2), \mathrm{Sn}_{9} \mathrm{M}(\mathrm{x} 1)\right)$ clusters and their structural parameters are gathered in Table 2D. Two yellow -orange $\mathrm{Sn}_{5} \mathrm{O}$ s clusters $[45,46]$ which contain well separated $\mathrm{NE}_{4}{ }^{+}(\mathrm{E}=\mathrm{Me} / 45 /$ or $\mathrm{H}$ 146/) cations and $\left[\mathrm{Os}\left(\mathrm{SnCl}_{3}\right)_{5} \mathrm{Cl}\right]^{3-}$ anions $/ 45,46 /$ are isostructural. The cluster anion has a central $\mathrm{Os}(\mathrm{II})$ atom coordinated to five $\mathrm{SnCl}_{3}$ moieties and a $\mathrm{Cl}$ atom (Fig. 6). Each Os(II) atom is octahedrally coordinated $\left(\mathrm{OsClSn}_{5}\right)$. The $\mathrm{Sn}-\mathrm{Os}$ bond distances range from $2.530(5)$ to $2.589(5) \AA$ in one molecule and 2.530(5) to 2.594(5) $\AA$ in the other. Each $\mathrm{Sn}(\mathrm{II})$ atom has a quasi - tetrahedral environment ( $\left.\mathrm{SnCl}_{3} \mathrm{Os}\right)$.
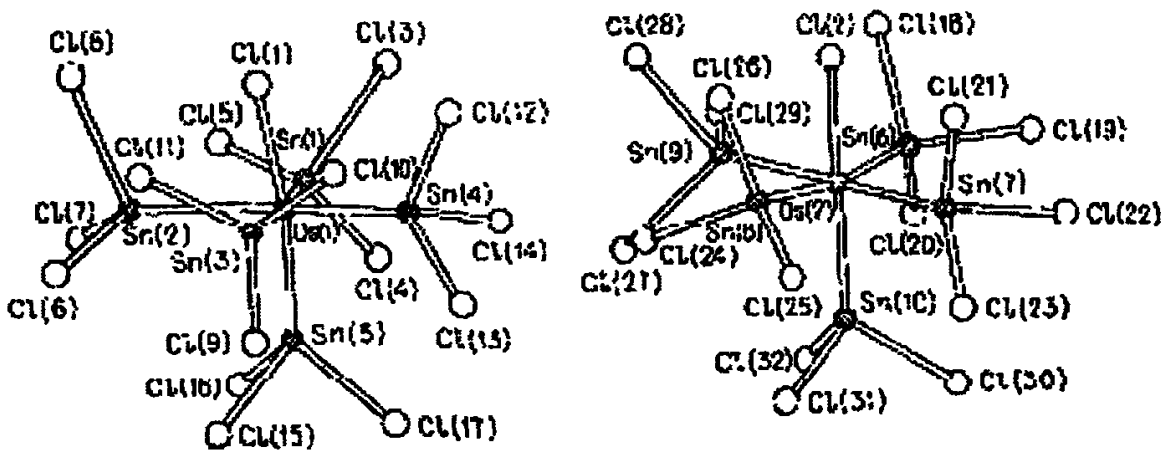

Fig. 6: Structure of $\left[\mathrm{Os}\left(\mathrm{SnCl}_{3}\right)_{5} \mathrm{Cl}\right]^{4-} / 46 /$

A $\mathrm{Sn}_{4}$ cluster $/ 47 /$ contains two structurally similar but symmetry independent molecules in a unit cell. Each has a crystallographically imposed centre of symmetry located in the middle of the sixteen membered ring, which is formally a [-O-Wcp*(O)-O-SnMe $\left.{ }_{3}-\right]_{4}$ tetramer. The interplanar angle between the $\mathrm{Sn}$ and $\mathrm{W}$ planes in one molecule is $11^{\circ}$ and in the other $14^{\circ}$. Each Sn atom has a trigonal bipyramidal arrangement $\left(\mathrm{SnC}_{3} \mathrm{O}_{2}\right)$, and each $\mathrm{W}$ atom has a typical three-legged piano-stool geometry $\left(\mathrm{WO}_{3} \mathrm{cp}{ }^{*}\right)$.

The structure of orange red $\left[\left\{\mathrm{cp}(\mathrm{CO})_{2} \mathrm{Fe}\right\}_{4} \mathrm{Sn}_{4} \mathrm{Se}_{6}\right]$ cluster /48/ consists of an adamantate - like $\mathrm{Sn}_{4} \mathrm{Se}_{6}$ framework (Fig. 7). Each $\mathrm{Sn}$ atom is bonded to a $\mathrm{Fe}(\mathrm{CO})_{2} \mathrm{Cp}$ unit with average $\mathrm{Sn}-\mathrm{Fe}$ bond distance of $2.507 \AA$ in one molecule and $2.522 \AA$ in the other. Each $\mathrm{Sn}$ atom has a quasi - tetrahedral environment $\left(\mathrm{SnSe}_{3} \mathrm{Fe}\right)$. 


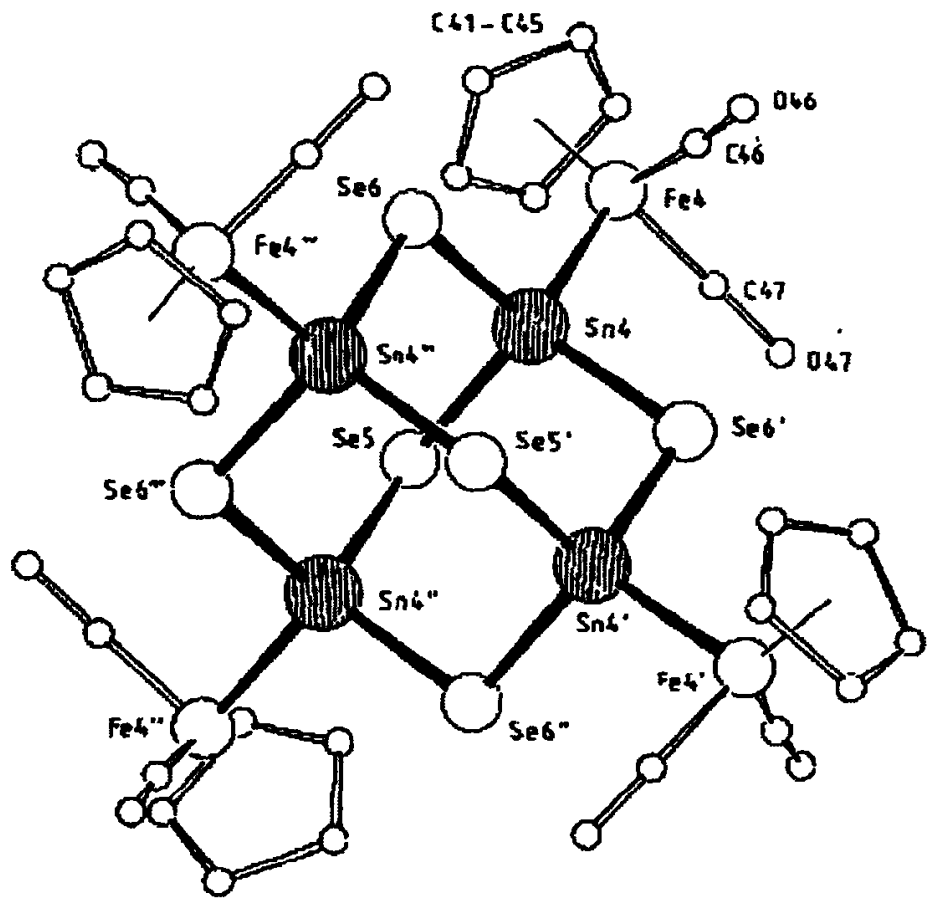

Fig. 7: Structure of $\left[\left\{\mathrm{cp}(\mathrm{CO})_{2} \mathrm{Fe}\right\}_{4} \mathrm{Sn}_{4} \mathrm{Se}_{6}\right] / 48 /$

A black cluster contains $[\mathrm{K}(\mathrm{cryp})]^{+}$cations and closo-[ $\left.\mathrm{Sn}_{9} \mathrm{Cr}(\mathrm{CO})_{3}\right]^{4-}$ anions $/ 49 /$. There are two crystallographically independent anions that are structurally identical in the unit cell, which reveals a virtually undistorted bicapped square antiprismatic shape (Fig. 8). The formation of the cluster can be viewed as the insertion of a neutral $\mathrm{Cr}(\mathrm{CO})_{3}$ fragment into the open square face of a side of a nido-Sn ${ }_{9}{ }^{4-}$ ion. The $\mathrm{Cr}(\mathrm{CO})_{3}$ fragment occupies a capping position in the bicapped square antiprism, with average $\mathrm{Sn}-\mathrm{Cr}$ bond length of 2.871(6) $\AA$. The $S n-S n$ distances within the square planes are 3.06(2) and 3.16(3) $\AA$. The distances between the two square - planes average at 2.99(2) $\AA$.
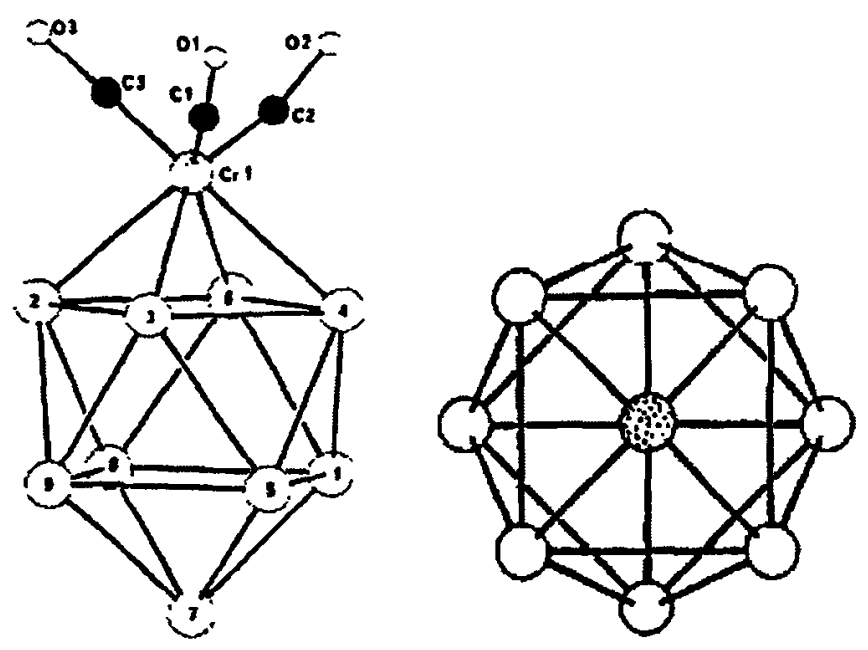

Fig. 8: Structure of $\left[\mathrm{Sn}_{9} \mathrm{Cr}(\mathrm{CO})_{3}\right]^{4-} / 49 /$ 


\section{CONCLUSION}

An analysis of over six hundred heterotin complexes from dimers to polymers shows that some $8 \%$ of them exist in isomeric forms. There are five derivatives /6-10/ which exist in two isomeric forms, one /11/ contains four crystallographically independent molecules, and the rest /6, 12-49/ contain two such molecules belonging to the class of distortion isomerism. In these the $\mathrm{Sn}$ atom is found in oxidation states of +2 (most common) and +4 with geometries including: trigonal planar $\left(\mathrm{SnO}_{3}, \mathrm{SnC}_{3}, \mathrm{SnP}_{3}, \mathrm{SnB}_{2} \mathrm{C}, \mathrm{SnPt}_{3}\right)$, tetrahedral $\left(\mathrm{SnN}_{3} \mathrm{Li}\right.$; $\mathrm{SnC}_{3} \mathrm{M}(\mathrm{M}=\mathrm{Li}, \mathrm{Si}, \mathrm{Ge}, \mathrm{Sn}, \mathrm{Pb}, \mathrm{Ti}, \mathrm{Mo}, \mathrm{Mn}, \mathrm{Fe}, \mathrm{Co}, \mathrm{Ni}, \mathrm{Ru}$ or $\mathrm{Pt})$; $\mathrm{SnCl}_{3} \mathrm{M}(\mathrm{M}=\mathrm{Mn}, \mathrm{Ru}$ or Pt); $\mathrm{SnSe}_{3} \mathrm{Fe} ; \mathrm{SnC}_{2} \mathrm{M}_{2}(\mathrm{M}=\mathrm{Fe}$, Co or $\mathrm{Re}) ; \mathrm{SnC}_{2} \mathrm{ORu} ; \mathrm{SnC}_{2} \mathrm{ClM}(\mathrm{M}=\mathrm{Mo}$ or $\mathrm{Fe}) ; \mathrm{SnClFe}_{2} \mathrm{Co} ; \mathrm{SnYbSn}_{3}^{\prime} ; \mathrm{SnSn}_{4}^{\prime}$ ), trigonal bipyramidal $\left(\mathrm{SnC}_{3} \mathrm{O}_{2}\right)$ and square pyramidal $\left(\mathrm{SnC}_{2} \mathrm{Br}_{2} \mathrm{Ir}, \mathrm{SnSn}_{4} \mathrm{Cr}, \mathrm{SnSn}_{5}\right)$.

The isomers crystallized in the following crystal classes: hexagonal $(\mathrm{x} 1)<$ tetragonal $(\mathrm{x} 2)<$ orthorhombic (x4) < triclinic (x18) < monoclinic (x24).

The heterometal atoms include transition and lanthanide metals along with non - transition metals. The mean Sn - M (non - transition) bond distance elongate in the order: $2.581 \AA$ (range 2.567(6) - 2.598(4) /14/, $\mathrm{Sn}-\mathrm{Ge})<2.828 \AA(2.809(2)-2.848(2) / 15 /, \mathrm{Sn}-\mathrm{Pb})<2.870 \AA(2.754(2) / 10 /-2.99(2) / 49 /, \mathrm{Sn}-\mathrm{Sn})<$ $2.900 \AA(2.861(7) / 12 /-2.97(5) / 13 /, \mathrm{Sn}-\mathrm{Li})<2.995 \AA(2.99,3.00 / 36 /, \mathrm{Sn}-\mathrm{Na})$. There is a much richer chemistry with the transition metals and the $\mathrm{Sn}-\mathrm{M}$ distances are summarized in Table 3 . As can be seen, iron subgroup elements and osmium generate a direct metal - metal bond with the mean distance less than $2.565 \AA$.

Table 3

Summary of the $\mathrm{Sn}-\mathrm{M}$ (transition) bond distances [ $\AA$ ] in the heterotin isomers

\begin{tabular}{c|c|c|c}
\hline & Shortest [ref.] & Longest [ref.] & Mean \\
\hline $\mathrm{Sn}-\mathrm{M}$ & & & \\
\hline$-\mathrm{Ni}$ & $2.541(1) / 22 /$ & $2.571(1) / 22 /$ & 2.556 \\
\hline$-\mathrm{Co}$ & $2.441(1) / 30 /$ & $2.681(1) / 44 /$ & 2.561 \\
\hline$-\mathrm{Os}$ & $2.530(1) / 46 /$ & $2.600(1) / 45 /$ & 2.562 \\
\hline$-\mathrm{Fe}$ & $2.490(5) / 48 /$ & $2.647(8) / 37 /$ & 2.563 \\
\hline$-\mathrm{Pt}$ & $2.345(1) / 7 /$ & $2.741(3) / 43 /$ & 2.619 \\
\hline$-\mathrm{Ru}$ & $2.571(1) / 42 /$ & $2.670(1 / 35 /$ & 2.620 \\
\hline$-\mathrm{Ir}$ & $2.636(1) / 23 /$ & $2.640(1) / 23 /$ & 2.638 \\
\hline$-\mathrm{Mn}$ & $2.575(5) / 19 /$ & $2.678(1) / 11 /$ & 2.646 \\
\hline$-\mathrm{Mo}$ & $2.513(3) / 40 /$ & $2.740(1) / 28 /$ & 2.667 \\
\hline$-\mathrm{Re}$ & $2.663(3) / 16 /$ & $2.874(3) / 40 /$ & 2.773 \\
\hline$-\mathrm{Ti}$ & $2.843(1) / 16 /$ & & 2.843 \\
\hline$-\mathrm{Cr}$ & $2.852(1) / 49 /$ & $2.890(1) / 49 /$ & 2.871 \\
\hline
\end{tabular}

This overview, together with its precursors $/ 1,2 /$ has surveyed over two hundred organotin compounds, serving to focus attention on area in the rich isomer chemistry of tin which could use additional study. There are four types of isomers: distortion, cis-trans, ligand and n-mer, of which the first one by far prevails $(\sim 98 \%)$. 


\section{ACKNOWLEDGEMENT}

The authors thank the Ministry of Education of the Slovak Republic, APVT 20005504 and VEGA 1/0353/08 for financial support.

\section{REFERENCES}

1. M. Melnik, J. Garaj, A. Valent, and M. Kohútová, The Icfai University Journal of Chemistry, 1 (2008) 36

2. M. Melník, J. Garaj, A. Valent, and M. Kohútová, Main Group Met. Chem., 32 (2009) 1.

3. C. E. Holloway and M. Melnik, Main Group Met. Chem., 24, (2001) 133.

4. C. E. Holloway and M. Melnik, Main Group Met. Chem., 24, (2001) 467.

5. M. Melnik, Coord. Chem. Rev., 47, (1982) 239.

6. M. J. Mc Geary, R. H. Cayton, K. Folting, J. C. Huffman, and K. G. Caulton, Polyhedron 11, (1992) 1369.

7. R. Willem, M. Gielen, G. Meunier - Piret, M.Van Meerssche, K. Jurschat, and A. Tzchach, J. Organomet. Chem. 277 (1984) 335.

8. S. Freitag, R. Herbst - Irmer, F. U. Richter, and H. Weichmann, Acta Crystallogr., Sect. C, 50 (1994) 1588.

9. M. P. Degaonkar, V. G. Puranik, S. S. Tavale, S. Gopinathan, and C. Gopinathan, Bull. Chem. Soc. Jpn., 67 (1994) 1797.

10. L.N. Bochkarev, O.V. Grachev, Molosnova N.E. and Zhiltsopv S.F., J. Organomet. Chem., 443 (1993) C26.

11. H. P. Weber and R. F. Bryan, Acta Crystallogr., 22 (1967) 822.

12. D. Reed, D. Stalke, and D. S. Wright, Angew. Chem. Int. Ed. Engl., 30 (1991) 1459.

13. K. W. Hellmann, L. H. Gade, O. Gevert, P. Steinert, and J. W. Lauher, Inorg. Chem., 34 (1995) 4069.

14. L. Párkányi, A. Kalmán, K. H. Pannel, and H. Sharma, J. Organomet. Chem., 484 (1994) 153.

15. N. Kleiner and M. Dräger, J.Organomet. Chem., 270 (1984) 151.

16. W. Zheng and D. W. Stephan, Inorg. Chem., 27 (1988) 2386.

17. A. N. Protsky, B. M. Bulychev, G. L. Soloveichik, and V. K. Belsky, Inorg. Chem. Acta, 115 (1986) 121.

18. F. Ettel, G. Hunter, L. Zsolnai, and C. Emmerich, J. Organomet. Chem., 414 (1991) 71.

19. S. Onaka, Bull. Chem. Soc. Japan, 48 (1975) 319.

20. M. Kay, K. M. Mackay, and B. K. Nicholson, J. Organomet. Chem., 431 (1995) 247.

21. H. F. Klein, K. Ellrich, D. Neugebauer, O. Orama, and K. Krüger, Z. Naturforsch., 38b (1983) 303.

22. S. Midollini, A. Orlandini, and L. Sacconi, J. Organomet. Chem., 162 (1978) 109.

23. A.A.H. van der Zeijden, G. van Koten, J. M. A. Wouters, W. F. A. Wijsmuller, D. M. Grove, W. J. J. Smeets, and A. L. Spek, J. Amer. Chem. Soc., 110 (1988) 5354.

24. C. J. Levy, J. J. Vittal, and R. J. Tuddephatt, Organometallics, 15 (1996) 2108.

25. E. Farkas, L. Kollár, M. Moret, and A. Sironi, Organometallics, 15 (1996) 1345. 
26. M. Veith, C. Ruloff, V. Huch, and F. Töllner, Angew. Chem.Int. Ed. Engl., 27 (1988) 1381.

27. M. Westerhausen, H. D. Hausen, and V. Schwarz, Z. Anorg. Allg. Chem., 621 (1995) 877.

28. V. K. Belsky, A. N. Protsky, B. M. Bulychev, and G. L. Soloveichik, J. Organomet. Chem., 280 (1985) 45.

29. M. Akita, T. Oku, M. Tanaka, and Y. Muro - Oka, Organometallics, 10 (1991) 3080.

30. O. Y. Curnov, B. K. Nicholson, and M. J. Severinsen, J. Organomet. Chem., 388 (1990) 379.

31. M. Westerhausen, R. Lőw, and W. Schwarz, J. Organomet. Chem., 513 (1996) 213.

32. C. J. Cardin, D. J. Cardin. M. A. Convery, M. M. Devereux, B. Twamley, and J. Silver, J. Chem. Soc., Dalton Trans., (1996) 1145.

33. H. Wadepohl, H. Pritzkov, and W. Siebert, Organometallics, 2 (1983) 1899.

34. T. Imori, R. H. Heyn, T. Don Tilley, and A. L. Rheingold, J. Organomet. Chem., 493 (1995) 83.

35. M. L. Buil, M. A. Esteruelas, F. J. Lahoz, E. Onate, and L. A. Oro, J. Am. Chem. Soc., 117 (1995) 3619.

36. M. Veith and R. Rősler, Z. Naturforsch., 41b (1986) 1071.

37. C. J. Gilmore and P. Woodward, J. Chem. Soc., Dalton Trans., (1972) 1387.

38. S. Sharma, J. Cervantes, J. L. Mata - Mata, M. C. Brun, F. Cervantes - Lee, and K. H. Pannel, Organometallics, 14 (1995) 4269.

39. J. Weaver and P. Woodward, J. Chem. Soc., Dalton Trans., (1973) 1060.

40. B. T. Huie, S. W. Kirtley, C. B. Knobler, and H. D. Kaesz, J. Organomet. Chem., 213 (1981) 45.

41. J. M. Cassidy and K. H. Whitmire, Inorg. Chem., 28 (1989) 2494.

42. R. D. Adams and D. A. Katahira, Organometallics, 1 (1982) 53.

43. R. Usón, J. Forniés, M. Tomás, and I. Usón, Angew. Chem. Int. Ed. Engl., 29 (1990) 1449.

44. M. Moll, H. Behrens, P. Mehrbach, K. Görting, G. Liehr, and R. Böhme, Z. Naturforsch., 35b (1980) 1115.

45. E. N. Yurchenko, E. T, Devyatkina, T. S. Khodashova, M. A. Porai - Koshits, V. I. Komnov, V. A. Varnek. P. G. Antonov, and Y. M. Kukushkin, Koord. Khim., 5 (1979) 552 ; Engl. Ed., p. 429.

46. T. S. Khodashova, M. A. Porai - Koshits, V. P. Nikolaev, and V. N. Shchurkina, Zh. Strukt. Khim, 21 (1980) 123; Engl. Ed., p. 220.

47. M. S. Rau, C. M. Kutz, G. L. Geoffrey, A. L. Rheingold, and B. S. Haggerty, Organometallics, 13 (1994) 1624.

48. K. Merzweil and L. Weisse, Z. Naturforsch., $45 b$ (1990) 971.

49. B. W. Eichborn, R. C. Haushalter, and W. T. Pennington, J. Amer. Chem. Soc., 110 (1988) 8704. 